Draft version December 16, 2016

Preprint typeset using $\mathrm{LAT}_{\mathrm{E}} \mathrm{X}$ style emulateapj v. 01/23/15

\title{
THE BURRELL SCHMIDT DEEP VIRGO SURVEY: TIDAL DEBRIS, GALAXY HALOS, AND DIFFUSE INTRACLUSTER LIGHT IN THE VIRGO CLUSTER
}

\author{
J. Christopher Mihos ${ }^{1}$, Paul Harding ${ }^{1}$, John J. Feldmeier ${ }^{2}$, Craig Rudick ${ }^{3}$, \\ Steven Janowiecki ${ }^{4}$, Heather Morrison ${ }^{1}$, ${\text { Colin } \text { Slater }^{5} \text { and Aaron Watkins }}^{1}$ \\ Draft version December 16, 2016
}

\begin{abstract}
We present the results of a deep imaging survey of the Virgo cluster of galaxies, concentrated around the cores of Virgo subclusters A and B. The goal of this survey was to detect and study very low surface brightness features present in Virgo, including discrete tidal features, the faint halos of luminous galaxies, and the diffuse intracluster light (ICL). Our observations span roughly 16 degrees $^{2}$ in two filters, reaching a $3 \sigma$ limiting depth of $\mu_{B}=29.5$ and $\mu_{V}=28.5 \mathrm{mag} \operatorname{arcsec}^{-2}$. At these depths, our limiting systematic uncertainties are astrophysical: variations in faint background sources as well as scattered light from galactic dust. We show that this dust-scattered light is well-traced by deep farinfrared imaging, making it possible to separate it from true diffuse light in Virgo. We use our imaging to trace and measure the color of the diffuse tidal streams and intracluster light in the Virgo core near M87, in fields adjacent to the core including the M86/M84 region, and to the south of the core around M49 and subcluster B, along with the more distant $\mathrm{W}^{\prime}$ cloud around NGC 4365. Overall, the bulk of the projected ICL is found in the Virgo core and within the $\mathrm{W}^{\prime}$ cloud; we find little evidence for an extensive ICL component in the field around M49. The bulk of the ICL we detect is fairly red in color $(B-V=0.7-0.9)$, indicative of old, evolved stellar populations. Based on the luminosity of the observed ICL features in the cluster, we estimate a total Virgo ICL fraction of $7-15 \%$. This value is somewhat smaller than that expected for massive, evolved clusters, suggesting that Virgo is still in the process of growing its extended ICL component. We also trace the shape of M87's extremely boxy outer halo out to $\sim 150 \mathrm{kpc}$, and show that the current tidal stripping rate from low luminosity galaxies is insufficient to have built M87's outer halo over a Hubble time. We identify a number of previously unknown low surface brightness structures around galaxies projected close to M86 and M84. The extensive diffuse light seen in the infalling $\mathrm{W}^{\prime}$ cloud around NGC 4365 is likely to be subsumed in the general Virgo ICL component once the group enters the cluster, illustrating the importance of group infall in generating intracluster light. Finally, we also identify another large and extremely low surface brightness ultra-diffuse galaxy, likely in the process of being shredded by the cluster tidal field. With the survey complete, the full imaging dataset is now available for public release.
\end{abstract}

Keywords: galaxies: clusters: individual (Virgo) — galaxies: individual (M87, M49, NGC 4365) galaxies: interactions - techniques: photometric

\section{INTRODUCTION}

The assembly of galaxy clusters is a hierarchical process, wherein massive clusters grow over time through the accretion of smaller groups and clusters of galaxies. As such, it is a messy process. In the low velocity dispersion group environment, individual galaxies interact and merge with one another, creating discrete tidal tails and plumes of material expelled from the galaxies by the tidal forces involved. As these groups then merge to form clusters of galaxies, more material is stripped from the galaxies, and the previously-liberated tails and plumes

\footnotetext{
mihos@case.edu, paul.harding@case.edu, jjfeldmeier@ysu.edu craigrudick@gmail.com, steven.janowiecki@uwa.edu.au, heather@vegemite.case.edu, ctslater@uw.edu, aew54@case.edu

${ }^{1}$ Department of Astronomy, Case Western Reserve University, Cleveland, OH 44106, USA

2 Department of Physics and Astronomy, Youngstown State University, Youngstown, OH 44555, USA

3 University of Kentucky, Lexington, KY 40506, USA

${ }^{4}$ International Centre for Radio Astronomy Research (ICRAR), University of Western Australia, 35 Stirling Highway, Crawley, WA 6009, Australia

5 Department of Astronomy, University of Washington, Seattle, WA 98195, USA
}

are mixed into the cluster environment. Over time, the continued accretion of mass adds to this diffuse stellar component, building the intracluster light (ICL) of the growing cluster (for recent reviews, see Arnaboldi \& Gerhard 2010, Tutukov \& Federova 2011, Mihos 2015).

A large number of numerical simulations have detailed this complex behavior of intracluster star production (e.g., Willman et al. 2004, Sommer-Larsen et al. 2005, Conroy et al. 2007, Murante et al. 2007, Rudick et al. 2006, 2011, Puchwein et al. 2010, Martel et al. 2012, Watson et al. 2012, Contini et al. 2014, and Cooper et al. 2015). Taken together, these simulations imply that ICL production is an inevitable consequence of hierarchical structure formation, and has implications in many areas of extragalactic astronomy, including the assembly of the cluster red sequence, the evolution of large elliptical galaxies, the metallicity of the intracluster medium, and the overall baryon fraction of galaxy clusters.

However, while the ICL is an important stellar component to study, historically it has proved difficult to detect, due to its low surface brightness, which is significantly fainter than the brightness of the night sky. Early observations (e.g., de Vaucouleurs \& de Vaucouleurs 1970, 
Melnick et al. 1977, Thuan \& Kormendy 1977, Struble 1988, Vilchez-Gomez et al. 1994, Bernstein et al. 1995) were able to detect the ICL, but were unable to place strong constraints on its spatial distribution and total luminosity. In the last decade, deeper imaging of galaxy clusters has clearly shown the ICL in a wide variety of environments (Feldmeier et al. 2002, 2004b, Lin \& Mohr 2004, Gonzalez et al. 2005, Zibetti et al. 2005, Krick \& Bernstein 2007, Toledo et al. 2011, Arnaboldi et al. 2012). These observations have shown both the smoother diffuse light that is centrally distributed, as well as the tidal tails and plumes from recent galaxy interactions in these galaxy clusters. At higher redshifts, the rapid $(1+z)^{4}$ cosmological surface brightness dimming makes it hard to study the ICL at the faintest levels, but recent observations have begun to probe clusters at intermediate redshifts of $z=0.5-1.0$ (Guennou et al. 2012, Burke et al. 2012, 2015, Presotto et al. 2014, Montes \& Trujillo 2014, Giallongo et al. 2014). Connecting ICL properties at high redshift to those observed locally will yield important constraints on cluster assembly and evolution over cosmic time.

Aside from the diffuse starlight itself, many individual intracluster objects have been detected in nearby galaxy clusters, including intracluster globular clusters (e.g., Williams et al. 2007a, Lee et al. 2010, Peng et al. 2011, Durrell et al. 2014), intracluster red giant stars (e.g., Ferguson et al. 1998, Caldwell 2006, Williams et al. 2007b), supergiant stars (Ohyama \& Hota 2013), and planetary nebulae (e.g., Arnaboldi et al. 1996, 2002, Feldmeier et al. 2004a, Gerhard et al. 2007, Castro-Rodriguez et al. 2009, Ventimiglia et al. 2011, Longobardi et al. 2015b), intracluster novae (Neill et al. 2005) and supernovae (Gal-Yam et al. 2003, Dilday et al. 2010, Sand et al. 2011, Barbary et al. 2012), and intracluster H II regions (Gerhard et al. 2002, Cortese et al. 2004, Sun et al. 2010). These discrete tracers of the intracluster light allow for a more detailed view of the stellar populations and formation history of the ICL, through studies of the metallicity, age, and kinematics of the individual sources.

Unfortunately, connecting the wide-field view of the deep ICL imaging studies at low and high redshift to the detailed but narrower probes of nearby clusters using individual intracluster tracers has proved difficult. Quantitative comparisons are often plagued by systematic uncertainties between the various observational techniques (see e.g., Williams et al. 2007b and Mihos et al. 2009 for examples), as well as the ambiguity in creating a clean operational definition of ICL that can be used in all observed cases and can be directly compared with theoretical studies (Rudick et al. 2011 and Cooper et al. 2015). To bridge these gaps, detailed observations of nearby galaxy clusters using both deep surface photometry and imaging of discrete ICL populations are needed, along with kinematic followup of the discrete tracers to connect the dynamical properties of the ICL back to computational simulations of cluster evolution.

The Virgo Cluster of galaxies is a prime target for such studies. Given its close proximity $(\approx 16.5 \mathrm{Mpc}$; Mei et al. 2007), moderate richness (Girardi et al. 1994), and wellstudied structure (Binggeli et al. 1987), Virgo is an ideal place to search for ICL, and many detections of individual intracluster objects have been made there. There is also a wealth of observational data on the Virgo cluster from many surveys over a large number of wavelengths (see the review in Ferrarese et al. 2012), including optical surveys such as the Virgo Cluster Catalog (VCC; Binggeli et al. 1985) and the Sloan Digital Sky Survey (SDSS; York et al. 2000), far-infrared studies from re-calibrated IRAS data (Miville-Deschenes \& Lagache 2005) and the more recent Herschel Virgo Cluster Survey (HeViCS; Davies et al. 2010, 2012), deep UV imaging from GALEX (Boselli et al. 2011), radio continuum from the VLA FIRST and NVSS surveys (Becker et al. 1995 and Condon et al. 1998) , 21-cm HI mapping from ALFALFA (Giovanelli et al. 2005), and ROSAT coverage of the cluster in X-ray (Böhringer et al. 1994). Most recently, the Next Generation Virgo Cluster Survey (Ferrarese et al. 2012) has performed a detailed optical survey of $104 \mathrm{deg}^{2}$ of the Virgo cluster in the $u^{*}$ griz photometric bands, providing a rich dataset for connecting the large scale distribution of Virgo's diffuse ICL to the cluster's galaxy and globular cluster populations.

The overall structure of the Virgo cluster was originally mapped out in detail by Binggeli et al. (1987, and references therein), building on earlier work by others (e.g., Shapley \& Ames 1930; de Vaucouleurs 1961). In their study, Binggeli et al. (1987) noted that Virgo had a complex structure, with two major subclusters: subcluster A, centered around the giant elliptical M87, and subcluster $\mathrm{B}$ to the south, centered around the giant elliptical M49. A number of smaller subclusters were also identified, including subcluster $\mathrm{C}$, surrounding the elliptical galaxies M59 and M60, along with the M, W, and $\mathrm{W}^{\prime}$ "clouds" of galaxies. This rich and complex dynamical structure argues that Virgo continues to assemble today, making it an ideal target for studying the on-going generation of intracluster light across a variety of local environments.

However, one of Virgo's strongest advantages for work like this - its close proximity - also makes deep surface photometry of the cluster a challenge. In order to reach surface photometry depths of $1 \%$ of the dark night sky $\left(\mu_{V} \sim 27 \mathrm{mag} \operatorname{arcsec}^{-2}\right)$ over angular scales of several degrees requires a telescope that delivers an extremely wide field of view, along with well-controlled flat-fielding and minimal scattered light. Furthermore, the observational demands of mapping a cluster like Virgo that spans such a large area of the sky typically requires a large amount of observing time on a telescope dedicated to the task. As a result, very few attempts have been made to map the low surface brightness features across the Virgo cluster on large angular scales. Early studies employed photographic imaging (e.g., Malin 1979; Malin \& Hadley 1999; Katsiyannis et al. 1998) and revealed clear signs of tidal debris around many of the luminous galaxies of Virgo, but these studies were largely qualitative, due to the difficulty in photometric calibration of photographic plates.

To rectify this situation and provide deep wide-field mapping of the Virgo cluster, we have conducted a survey of diffuse light in Virgo using Case Western Reserve University's 0.6/0.9m Burrell Schmidt telescope. The wide field of view and closed-tube design of this telescope makes it ideal for deep surface photometry over large areas, and we have made extensive upgrades to the telescope and detectors to further improve its imaging capabilities. Our main goals for the survey, which 
spanned the years 2004-2011, were to detect and measure the properties of Virgo's diffuse ICL across the main subclusters $\mathrm{A}$ and $\mathrm{B}$, including individual tidal streams around galaxies as well as the more diffuse cluster ICL and stellar halos surrounding its massive ellipticals. The survey used two photometric passbands, delivering accurate surface photometry down to a $3 \sigma$ limiting surface brightness of $\mu_{V}=28.5 \mathrm{mag} \operatorname{arcsec}^{-2}$ and $B-V$ colors to $\mu_{V}=27.5 \mathrm{mag} \operatorname{arcsec}^{-2}$. A variety of results from our survey have been published in earlier papers, including the original deep imaging of ICL in the Virgo core (Mihos et al. 2005), a comparison between the diffuse ICL and intracluster planetaries (Mihos et al. 2009), color mapping of the core ICL (Rudick et al. 2010), a study of accretion signatures in Virgo ellipticals (Janowiecki et al. 2010), a photometric study of the halo of M49 (Mihos et al. 2013a), and the discovery of large and extremely low surface brightness ultra-diffuse galaxies (UDGs) in Virgo (Mihos et al. 2015).

In this paper, we present the imaging over the full area of the Burrell Schmidt survey in both photometric bands, and give a detailed report on a variety of low surface brightness features not yet reported elsewhere. In Section 2, we outline the survey's observational strategy and data reduction techniques (with complete details described more thoroughly in the accompanying Appendix). Section 3 describes our methodology for discriminating between Virgo intracluster light and a significant source of contamination in deep imaging - diffuse structure arising from scattering of Milky Way starlight off of foreground Galactic dust. Section 4 describes the properties of the diffuse starlight found in a variety of fields throughout Virgo, including areas around M87 and M86 in subcluster A, around M49 in subcluster B, and around NGC 4365 in the $\mathrm{W}^{\prime}$ cloud. We also report the discovery of a new Virgo ultradiffuse galaxy, likely in the process of being tidal shredded by Virgo's tidal field. Finally, in Section 5, we discuss our findings in the context of Virgo's overall dynamical evolution. Throughout this paper, we adopt a mean distance to Virgo of $16.5 \mathrm{Mpc}$ (Mei et al. 2007); at this distance $1^{\prime \prime}$ subtends 80 parsecs, while $1^{\prime}$ subtends 4.8 kiloparsecs. We also report all magnitudes in the Vega system, and give surface brightnesses in units of mag $\operatorname{arcsec}^{-2}$.

\section{OBSERVATIONS AND DATA REDUCTION}

Here we describe briefly our observing and data reduction strategies; the interested reader can find a more thorough description of these issues in the Appendix. The imaging was taken over the course of 107 nights, during seven observing seasons from 2004-2011, using the 24/36 inch Burrell Schmidt (hereafter, the Burrell) at KPNO. The telescope's well-baffled, closed tube design, its aggressively anti-reflection coated filters and dewar window, and the wide field of view of its CCD imager (originally $0.825^{\circ} \times 1.65^{\circ}$, now $1.65^{\circ} \times 1.65^{\circ}$ ), all make the telescope ideal for performing deep wide-field surface photometry, and we have made a number of modifications to the telescope/camera system over the course of the project to take advantage of these strengths. The telescope used a SITe ST-002A $2048 \times 4096$ pixel CCD $\left(0.825^{\circ} \times 1.65^{\circ}\right)$ during the first four observing seasons, which was later upgraded to a STA0500A 4096×4096 pixel CCD from the University of Arizona's Imaging
Technology Lab $\left(1.65^{\circ} \times 1.65^{\circ}\right)$ for the last three seasons. Both CCDs had $15 \mu \mathrm{m}$ pixels, yielding pixel scales of 1.45 arcsec pixel ${ }^{-1}$.

Our imaging was taken through two filters: Washington $M$, similar to Johnson $V$ but $\sim 250 \AA$ bluer (which cuts out variable night sky emission lines in the red), along with a modified (slightly bluer) Johnson $B$. We chose the filters to balance the signal of the ICL (likely peaking in the red due to its expected old stellar populations) with the noise of the sky (reduced in the blue due to the absence of variable night sky emission lines). To further reduce sky noise and scattered light from the moon and neighboring cities, we conducted imaging only under moonless photometric conditions where the variation in frame-by-frame photometric zeropoints (after correction for airmass and nightly zeropoint changes) was typically 0.01 magnitudes. During each season, a particular portion of the Virgo Cluster was targeted, and a series of 30-100 images of the field was taken in an individual filter, with the images dithered by up to half a degree relative to one another. Flat fielding was achieved by taking a series of $\sim 30-70$ night sky images bracketing the Virgo exposures in time and hour angle, and combining these images to produce a "super sky flat" for each season. Exposure times for both the Virgo fields and the blank night skies were 900 s in $M$ and 1200 s in $B$, yielding sky backgrounds of 900-1500 ADU and 700-1000 ADU in $M$ and $B$, respectively. A total of 461 Virgo images were taken in $M$ over the first five seasons, and 173 images in $B$ over the final two seasons (with a comparable number of blank sky images for flat fielding). A log of observations is given in Section A.2 and Table 1 of the Appendix.

With the data obtained, we then began the process of carefully reducing each image, registering each image spatially, and combining the images into a stacked mosaic image. We did this using the IRAF ${ }^{6}$ computing environment and our own software. During data reduction, we flat field each image by its season's night sky flat; tests show that these night sky flats are accurate to better than $0.5 \%$. After flat fielding, we calculate the photometric solution for each image based on photometry of the $\approx 100-200$ bright $(g<16)$ stars from the Sloan Digital Sky Survey (SDSS; Data Release 9; Ahn et al. 2012) typically found in each field. By transforming the SDSS magnitudes to Johnson $B$ and $V$ using the prescription of Lupton (described in the Appendix), our final mosaics are tied to the standard Johnson system, and we hereafter refer to the data as Johnson $B$ and $V$ imaging. From each reduced image, we next subtract the extended wings of bright stars using techniques described in Slater et al. (2009), followed by the subtraction of a sky plane, fit after masking bright stars and galaxies in the field using IRAF's objmasks task.

At this point, the individual images are all flattened and star- and sky-subtracted. For each filter, the images are then spatially registered (preserving the native $1.45^{\prime \prime}$ pixel $^{-1}$ image scale) and scaled in flux to a common photometric zero point. The individual images are

6 IRAF is distributed by the National Optical Astronomy Observatory, which is operated by the Association of Universities for Research in Astronomy (AURA) under cooperative agreement with the National Science Foundation. 
then median-combined to construct the final $B$ and $V$ mosaics, with obvious artifacts (bad columns, internal reflections, etc) masked during the process. At this point, we also correct the mosaics for the very slight residual background (1.125 ADU and 0.125 ADU in $B$ and $V$, respectively) still remaining. We also adopt a photometric solution for the mosaics which uses the common photometric zeropoint used in scaling the images for the median combine and a mean color term averaged over all seasons of the survey (see Table 2). Finally, as a last step in the data reduction, we re-run the masking algorithm on the final mosaics, then spatially re-bin the mosaics to increase signal to noise at the lowest surface brightness. The IRAF objmasks task is used to mask high surface brightness sources such as stars, background galaxies, and the inner regions of bright galaxies. We then rebin the masked mosaics into $9 \times 9$ pixel $\left(13^{\prime \prime} \times 13^{\prime \prime}\right)$ bins, calculating the median value in each spatial bin. Bins with more than half their pixels masked are themselves masked.

Assessing photometric depth for surface photometry is complicated, as it depends on a variety of noise sources that operate on different spatial scales, such as uncertainty in flat fielding and sky subtraction, the proximity of bright stars and galactic dust (see Section 2), and the spatial distribution of contaminants such as faint stars and background galaxies. We assess our limiting surface brightness in a number of ways. First, the variance in $9 \times 9$ pixel intensities measured in blank regions of the mosaics is 1.2 ADU in both our $B$ and $V$ mosaics, leading to a $3 \sigma(1 \sigma)$ limits of $\mu_{B}=27.5(28.7)$ and $\mu_{V}=27.1$ (28.3). However, this is a pessimistic limit, since surface photometry is typically done over large spatial scales averaging over many pixels. The variation in median intensity measured in $1^{\prime}$ boxes clustered in $10^{\prime}$ regions (which measures uncertainty due to local background contaminants) is much smaller: $0.25 \mathrm{ADU}$ in both mosaics, giving $3 \sigma(1 \sigma)$ limits of $\mu_{B}=29.4(30.6)$ and $\mu_{V}=28.6(29.8)$. Finally, a measure of the variation in median intensity measured in $10^{\prime}$ regions scattered across the mosaic a measure of global sky uncertainty - is $0.2 \mathrm{ADU}(B)$ and $0.4 \operatorname{ADU}(V)$, similar to the local background uncertainty. While the photometric limits for any individual object measured from these images will depend on its size and local background, as a fiducial number for expressing the limiting surface brightness of the survey, we adopt characteristic $3 \sigma$ limits of $\mu_{B, l i m}=29.5$ and $\mu_{V, \text { lim }}=28.5$

Our deep, binned Virgo mosaics are shown in Figures 1 and 2. In these images, the core of the cluster appears in the upper (northern) region, with M87 near the center, M89 to the far east (left) of the image, and the M86/M84 pair at the far west (right) of the image. The lower (southern) region shows the bright elliptical M49 near the center, with NGC 4365 to the southwest (lower right) and NGC 4535 to the east (left). Because of our large scale dithering, the exposure time varies across the mosaic; in the central regions, near M87 and M49 (the core of the Virgo A and B subclusters respectively), the total exposure times are typically 20 hours, but the exposure time drops towards the edges of the mosaic. The median exposure time for the mosaic is 7.75 hours, and Figure 1 only shows regions where we have more than 5 images (1.25 hours) contributing to the mosaic. At this threshold, the $V$ mosaic covers 16.7 square degrees. Our deep $B$ mosaic (Figure 2a) surveys 15.3 square degrees with at least 5 images (1.67 hours) contributing to the mosaic; the median exposure time is 7.7 hours, while the inner regions have typical exposure times of 18 hours.

Figure $2 \mathrm{p}$ shows the $B-V$ color map of the Virgo core where again we restrict the map to those areas that are covered with at least 5 images in both $B$ and $V$. The total area covered in this color map is 11.1 square degrees, and the image only shows colors in regions with surface brightness brighter than $\mu_{V}=28.5$, since at lower surface brightness the noise in the colors becomes extreme.

\section{DETECTING CONTAMINATION FROM GALACTIC CIRRUS}

Figures 1 and 2 show a great deal of diffuse light spread throughout the Virgo Cluster. However, it is likely that much of this light is not true intracluster light in the Virgo cluster, but galactic light backscattered from dust in the disk of our own galaxy, the so-called "galactic cirrus" (Low et al. 1984; see also the discussion by Sandage 1976). Galactic cirrus apears in the images as a diffuse, low surface brightness structure similar in many ways to the diffuse intracluster light we are searching for, and there is significant potential for confusion. Fortunately, in practice, two particular properties of the cirrus may be useful in distinguishing it from the ICL. First, with characteristic temperatures of $\mathrm{T}=7-20 \mathrm{~K}$ (e.g., Low et al. 1984, Finkbeiner et al. 1999, Veneziani et al. 2010), the cirrus radiates thermally in the far infrared, and can be detected in IRAS and Herschel maps. Second, since the optical light is scattered Milky Way starlight, it ought to be bluer on average than that typically expected for intracluster light (Rudick et al. 2010), although the presence or absence of extended red emission (ERE) in diffuse dust clouds can lead to a significant scatter in optical colors (Guhathakurta \& Tyson 1989, Witt et al. 2008, Brandt \& Draine 2012, Ieneka et al. 2013). Of course, the discriminatory power of the far infrared dust emission is not completely unambiguous. For example, one could envision stripping of young stars from a spiral galaxy yielding an ICL feature with blue colors and some associated dust emission.

Herschel imaging provides an ideal tool to probe the distribution of galactic cirrus in the Virgo field (Bianchi et al. 2016). At $250 \mu$, the SPIRE instrument provides a $18.1^{\prime \prime}$ beam imaged onto $6^{\prime \prime}$ pixels, comparable in spatial scale to our rebinned deep optical maps. In Figure 3 a we show the deep $250 \mu$ map of Virgo from the Herschel Virgo Cluster Survey (HeViCS; Davies et al. 2010; 2012), covering the same area as our deep optical images in Figures 1 and 2. The image is dominated by a ring-like distribution of dust in the northern half, with the core of Virgo being viewed through a fortuitous hole in the dust. The southern half of the image, extending down to fields surrounding M49, appears largely devoid of dust. On smaller scales, the $250 \mu$ map shows many features which correspond to features in our optical imaging, most noticeably in regions south of M89, southeast of M87, southwest of M84/M86, and in the northernmost part of the image.

Using the Herschel map, we can make a simple attempt to correct our optical image for this contamination from galactic cirrus. Studies of high-latitude, optically thin 

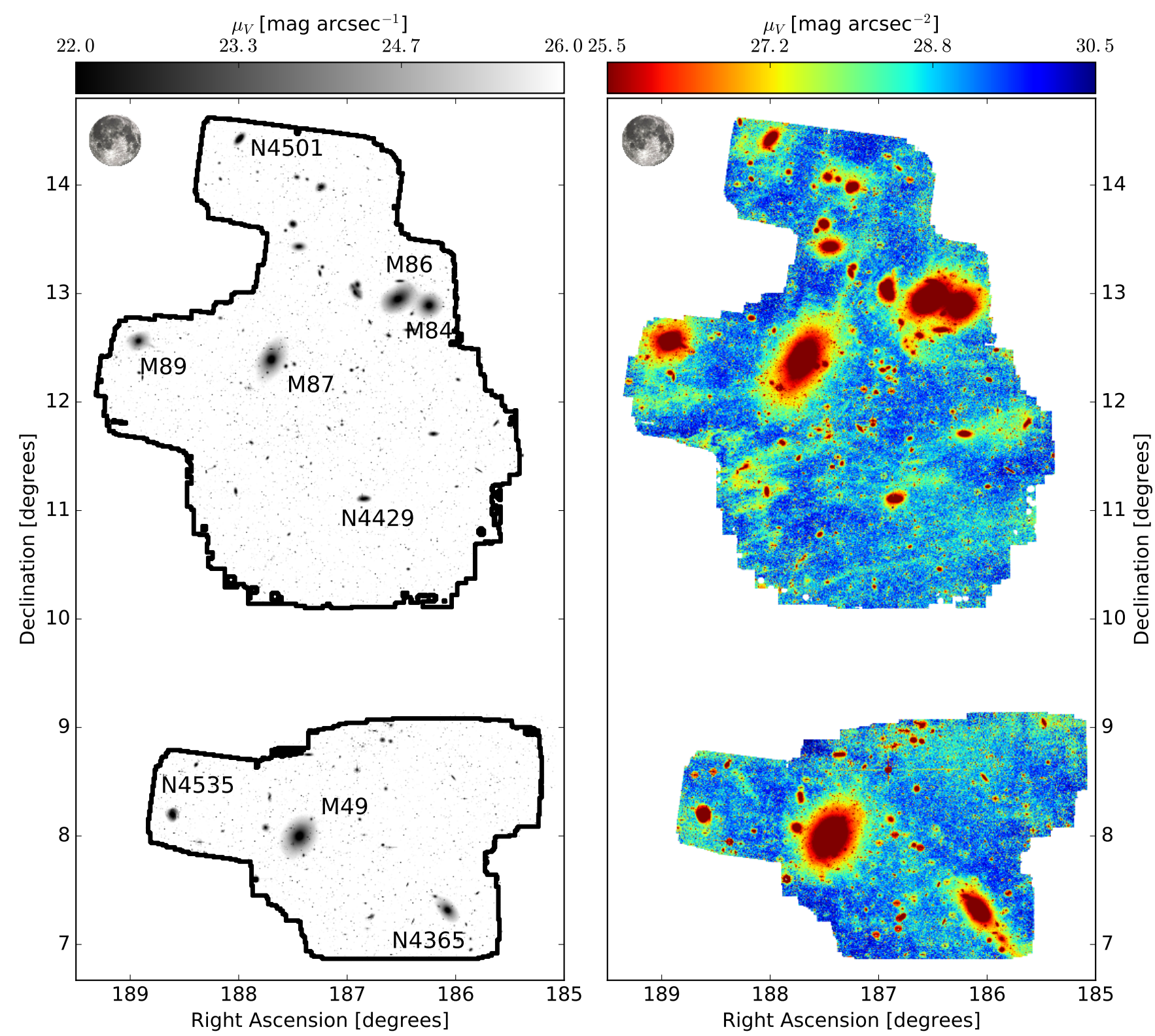

Figure 1. Final $V$ mosaic. The left panel shows a high surface brightness stretch of the V-band footprint of our Virgo survey, with several bright galaxies labeled. The right panel shows a low surface brightness stretch, where the extended halos of galaxies can be seen, along with diffuse intracluster light and galactic cirrus. North is up, east is to the left, and the full moon icon illustrates a half degree diameter scale (which projects to $145 \mathrm{kpc}$ at $\mathrm{d}=16.5 \mathrm{Mpc}$ ).

dust clouds (Witt et al. 2008, Ienaka et al. 2013) show a linear relationship between the optical flux and the IRAS $100 \mu$ emission. If such a model holds for the features in our deep optical imaging, we can attempt to remove the cirrus from our image by subtracting a suitably scaled version of the $250 \mu$ map from our $V$-band mosaic. We do this very simply, via trial-and-error subtraction using different scale factors for the $250 \mu$ map, until we achieve a satisfactory subtraction. Converting our surface brightnesses to linear flux densities (in MJy/sr) using the photometric transformation from Johnson to AB magnitudes given in Frei \& Gunn (1994), we find that a scale factor of $I_{\nu}(\mathrm{V}) / I_{\nu}(250 \mu)=3.5( \pm 0.5) \times 10^{-3}$ does a reasonable job of removing the cirrus without significantly oversubtracting the image. The resulting "cirrus-subtracted" optical map is shown in Figure 3 b.

The Herschel-subtracted map shows clearly that much of the diffuse structure we see in the raw optical maps comes from material with associated far-infrared emission - likely galactic cirrus. Nonetheless, many features remain, including nearly all the diffuse features in the core described by Mihos et al. (2005). A notable exception is the diffuse plume north of NGC 4435/8, which has previously been studied by Cortese et al. (2010) and shown to be associated not only with far-infrared flux but also HI at galactic velocities - all the signatures expected for galactic cirrus.

As a secondary check, we also use our unsubtracted optical maps to examine the colors of the cirrus in regions southeast of M87, where the cirrus dominates the 

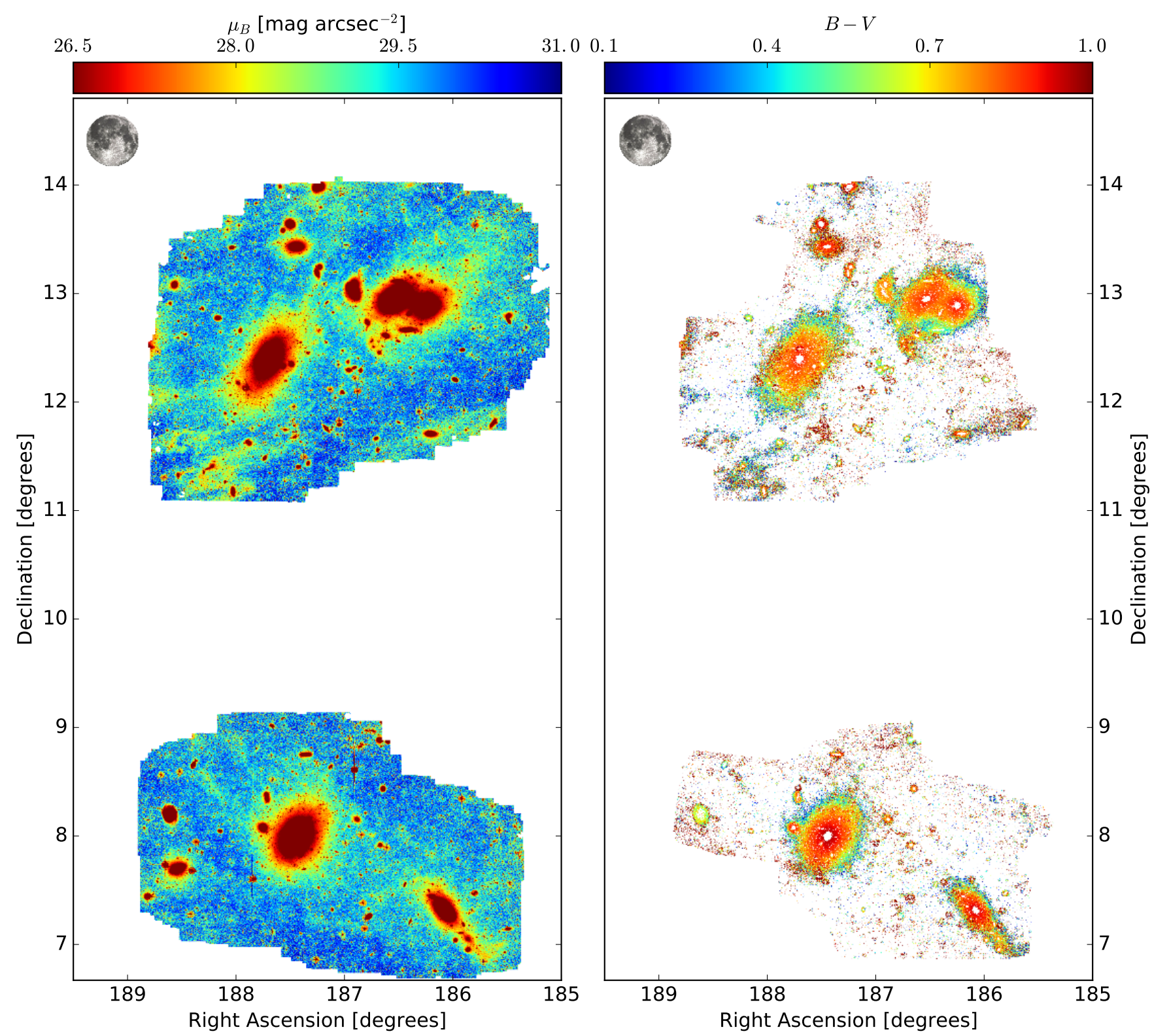

Figure 2. Left: final $B$ mosaic, stretched in intensity to show low surface brightness features. Right: $B-V$ colormap, showing pixel colors down to a surface brightness of $\mu_{V}=28.25$. North is up, east is to the left, and full moon shows a half degree diameter scale (144 $\mathrm{kpc}$ at $\mathrm{d}=16.5 \mathrm{Mpc})$.

low surface brightness structure. We use the differential photometry technique described in Rudick et al. (2010), wherein we lay down polygonal apertures at various spots in this region, and calculate the median pixel intensity values in each region after masking out discrete sources. We do the same for surrounding background fields to subtract off a local background level from the region photometry. Finally, we use the background-corrected region photometry to calculate the characteristic surface brightness and color of the regions. We find the $B-V$ color spans values from 0.4 to 0.8 , with typical uncertainties of several tenths (see Rudick et al. 2010 for a detailed discussion of the color uncertainties at low surface brightness). While galactic cirrus can span a wide range of optical colors (e.g., Witt et al. 2008), the $B-V$ colors we measure are indeed somewhat bluer than expected for the old stellar populations likely to characterize the bulk of the ICL in Virgo ( $B-V \sim 0.7-1.0$; Rudick et al. 2010), but by themselves do not discriminate against somewhat younger ICL populations. However, the combination of bluer colors and infrared emission are a likely signature of galactic cirrus.

\section{LOW SURFACE BRIGHTNESS STRUCTURES IN VIRGO}

In this section we identify and discuss low surface brightness features present in the data that do not have counterparts in the Herschel $250 \mu \mathrm{m}$ map — i.e., those that are unassociated with galactic cirrus, and likely to be diffuse light in Virgo itself. While a number of these features were identified in our previous imaging releases (Mihos et al. 2005 [M+05], Janowiecki et al. 2010 [J+10], Rudick et al. 2010, Mihos et al. 2013a), our wider imag- 

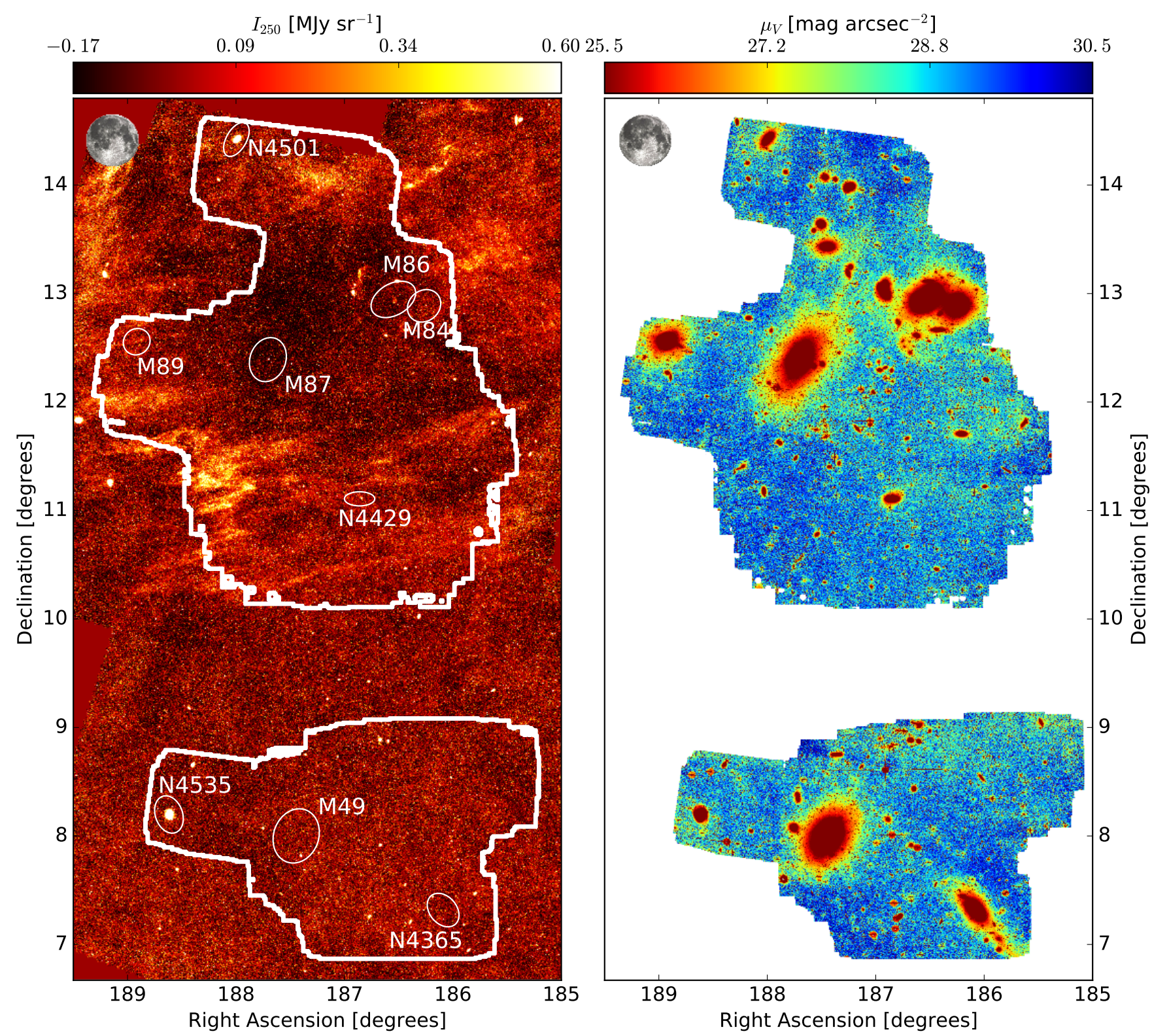

Figure 3. Left: Deep Herschel $250 \mu$ map of Virgo from the HeViCS project (Davies et al. 2010; 2012); the diffuse emission traces galactic cirrus. The white contour shows the areal coverage of our V-band imaging, and bright galaxies are marked. Right: A "dust corrected" view of Virgo, obtained by scaling and subtracting the HeViCS map from our V mosaic. See text for details. North is up, east is to the left, and full moon shows a half degree diameter scale (144 kpc at d=16.5 Mpc)..

ing area presented here has revealed a number of new features and also permits color measurements for several of the previously identified ones. In addition, detection in both the $B$ and $V$ mosaics - imaging taken across several observing seasons using different instrumental setups - gives us added confidence in identifying structures which were marginal detections using previously published data in a single mosaic.

In examining the deep mosiacs, a wide variety of low surface brightness features can be found on varying spatial scales, from the very extended streams emanating to the NW of M87 (M+05, Rudick et al. 2010, Figures 1 and 2 to a myriad of small LSB objects that span only a handful of pixels. We show in Figure 4 several examples of the variety of structures visible in our mo- saics. The left panel shows a $35^{\prime} \times 24^{\prime}$ field near the top of the southern survey area. Some of the LSB structures are associated with known background sources, including ICL in the distant background galaxy cluster Abell 1541 $(z=0.09$, Slinglend et al. 1998) and the tidal tails associated with the background galaxy group around the radio galaxy NGC $4410\left(c z=7254 \mathrm{~km} \mathrm{~s}^{-1}\right.$, Hummel et al. 1986, Donahue et al. 2002). We also trace the faint outer arms of the Virgo spiral NGC 4411 to a distance of $3.6^{\prime}(17.3 \mathrm{kpc})$. The right panel shows a $12^{\prime} \times 10^{\prime}$ field near the Virgo dwarf galaxy IC 3418. This tidally disturbed galaxy is in the process of being ram pressure stripped, and sports a streamer of star forming knots extending to the southeast (Hester et al. 2010, Kenney et al. 2014). To the east of IC 3418 is a small $\left(r=15^{\prime \prime}\right)$, 
uncatalogued nucleated LSB galaxy, while to the south, the background galaxy VPC $678(z=0.18)$ shows extended tidal tails. In these two cutouts, only NGC 4411 and IC 3418 are known Virgo galaxies; the uncatalogued LSB galaxy has no redshift and may either be one of the many LSB dwarfs in Virgo (e.g., Ferrarese et al. 2016) or a background source, while the other systems are all much more distant. These fields represent only $\sim 1 \%$ of the survey area, and similar features are seen throughout the rest of our data. Our intent in this paper is not to create any sort of complete surface brightness limited catalog of structures in Virgo, but instead to identify and discuss the properties of the large LSB structures that are most likely to trace dynamical events in the Virgo cluster. As such, for the remainder of this study, we focus on these larger LSB structures and leave the smaller features for later study.

For ease of discussion, we break up this section to focus on specific subregions of Virgo. In what follows, we use the Herschel map to discriminate between galactic cirrus and diffuse light in Virgo, but due to the quantitative uncertainty of the cirrus subtraction using the Herschel data, all photometric analysis is done on the unsubtracted optical maps alone; we simply avoid regions of cirrus contamination in our analysis. In describing the diffuse features we find in Virgo, we detail surface brightnesses and, where possible, measured $B-V$ colors. As mentioned previously, detailed error modeling at low surface brightness is complicated, and depends on the global sky uncertainty, the total extent of the ICL feature being measured, and the fluctuation in background sources on spatial scales comparable to that of the feature. For purposes of the discussion here, however, we note that typical photometric uncertainties in surface brightness are approximately $0.1 \mathrm{mag}$ at $\mu_{V}=28.5$, while $B-V$ colors can be probed to a similar $0.1 \mathrm{mag}$ uncertainty down to a surface brightness of $\mu_{V} \sim 27.5$. Finally, in describing the galaxies associated with each field, we give their observed radial velocities for context. Unless noted otherwise, these velocities are taken from the Sloan Digital Sky Survey DR12 (Alam et al. 2015).

\subsection{The M87 Field}

In the core of the Virgo Cluster, the field around the giant elliptical galaxy M87 (1284 $\mathrm{km} \mathrm{s}^{-1}$, Cappellari et al. 2011) hosts a rich assortment of diffuse stellar features. Many of these were detailed in our earlier papers, including M87's very extended and irregular stellar halo $(\mathrm{M}+05, \mathrm{~J}+10$; see also the discussion below) and the two long, linear streamers seen projecting to the northwest from M87 (streams A and B in the nomenclature of $\mathrm{M}+05$ ). A closer examination of this field using our updated mosaics shows that a number of the small galaxies in close vicinity to the giant elliptical also sport tidal features suggestive of ongoing tidal stripping. Figure 5 shows these structures - all of which can be seen in both the $B$ and $V$ mosaics - in more detail. Lying $\sim 38^{\prime}(182$ $\mathrm{kpc})$ southeast of M87, VCC $1426\left(833 \mathrm{~km} \mathrm{~s}^{-1}\right)$ shows a low surface brightness tail $\left(\mu_{V}=28.2, B-V=0.6\right)$ extending $3.9^{\prime}$ (19 kpc) to the south. VCC 1426 is part of a trio of small galaxies including IC $3461\left(993 \mathrm{~km} \mathrm{~s}^{-1}\right)$ and IC $3466\left(883 \mathrm{~km} \mathrm{~s}^{-1}\right)$ which all lie within $6.5^{\prime}$ of each other. The tight grouping in velocity of these galaxies suggests that they may be a physical group, in which case VCC 1426's tidal tail may come from slow interactions between the group members rather than by tidal stripping from the cluster potential or M87 itself. This stripping of material from galaxies as they interact on group scales inside the larger cluster environment can enhance the production of ICL in clusters over that drawn out by global tides and high speed encounters alone (e.g., Mihos 2004, Rudick et al. 2009).

Another set of streams can be seen $30^{\prime}$ (144 kpc) northwest of M87. One is a linear stream associated with VCC 1153 (844 km s ${ }^{-1}$, Rines \& Geller 2008), extending $5^{\prime} \mathrm{SW}$ of the galaxy. The stream has a surface brightness of $\mu_{V}=27.9$ and color of $B-V=0.7$. Projected very close to this system is VCC $1148\left(1417 \mathrm{~km} \mathrm{~s}^{-1}\right)$ with its own plume $\left(\mu_{V}=27.5, B-V=0.8\right)$ extending $1.6^{\prime} \mathrm{ENE}$. Unlike the situation in the VCC 1426 group, the relatively large velocity difference between these two galaxies makes it unlikely that these features arise from an interaction between these galaxies; instead, it is likely due to interactions with M87 or other galaxies in Virgo. Finally, $\sim 7^{\prime} \mathrm{SSW}$ of the galaxy pair there is a broad, low surface brightness plume $\left(\mu_{V}=27.5, B-V=0.8\right)$ without any obvious connection to other galaxies in the field.

The streams highlighted here all come from low luminosity companion galaxies and are themselves low luminosity streams. We also note one additional stream (marked with a small arrow in Figure 5), due south of M87 and extending outwards parallel to the minor axis of the galaxy. This stream has no clear association with any of M87's existing companions and is likely the remnant of a tidally shredded dwarf. With characteristic surface brightnesses of $\mu_{V} \sim 27.5-28.0$, and covering a few square arcminutes in size, these various tidal features contain only a few $\times 10^{7} \mathrm{~L}_{\odot, V}$ of starlight, in contrast to the much more extended plumes and streams identified by $\mathrm{M}+05$ which have larger luminosities of $\sim 10^{8} \mathrm{~L}_{\odot, V}$ $(\mathrm{J}+10)$.

While the luminosity of the tidal streams is relatively low, in the active dynamical environment that characterizes the core of the Virgo cluster, their lifetimes will be short as well, such that the implied rate of ICL production could yet be significant. Dynamical models of ICL evolution (Rudick et al. 2009) show that coherent streams only survive 1-2 crossing times before being disrupted, which corresponds to roughly $300 \mathrm{Myr}$ in the outer halo of M87 (at $r=75 \mathrm{kpc}$ ). With a total stream luminosity of $\sim 5 \times 10^{8} \mathrm{~L}_{\odot, V}$ (including the features identified in $\mathrm{J}+10$ ), if streams are continuously created and destroyed, this argues for an instantaneous (i.e., timescales $<0.5 \mathrm{Gyr}$ ) luminosity accretion rate from low mass satellites onto the M87 halo of $\sim 1$ $\mathrm{L}_{\odot, V} \mathrm{yr}^{-1}$. M87's halo contains appreciable luminosity even at these radii - integrating the measured luminosity profile $(\mathrm{J}+10)$ beyond $75 \mathrm{kpc}$ to the outermost measured point (at $\mathrm{r}=175 \mathrm{kpc}$ ) yields a total luminosity of $2 \times 10^{10} \mathrm{~L}_{\odot, V}$. This is significantly more than would be expected from the current accretion rate, arguing that infall of small satellites is not the dominant mechanism for building the outer halo of Virgo's central galaxy. This conclusion is consistent with models for the growth of $\mathrm{cD}$ galaxies, which show that central galaxies assemble late (at $z<1$ ) from mergers of more massive systems (e.g., 

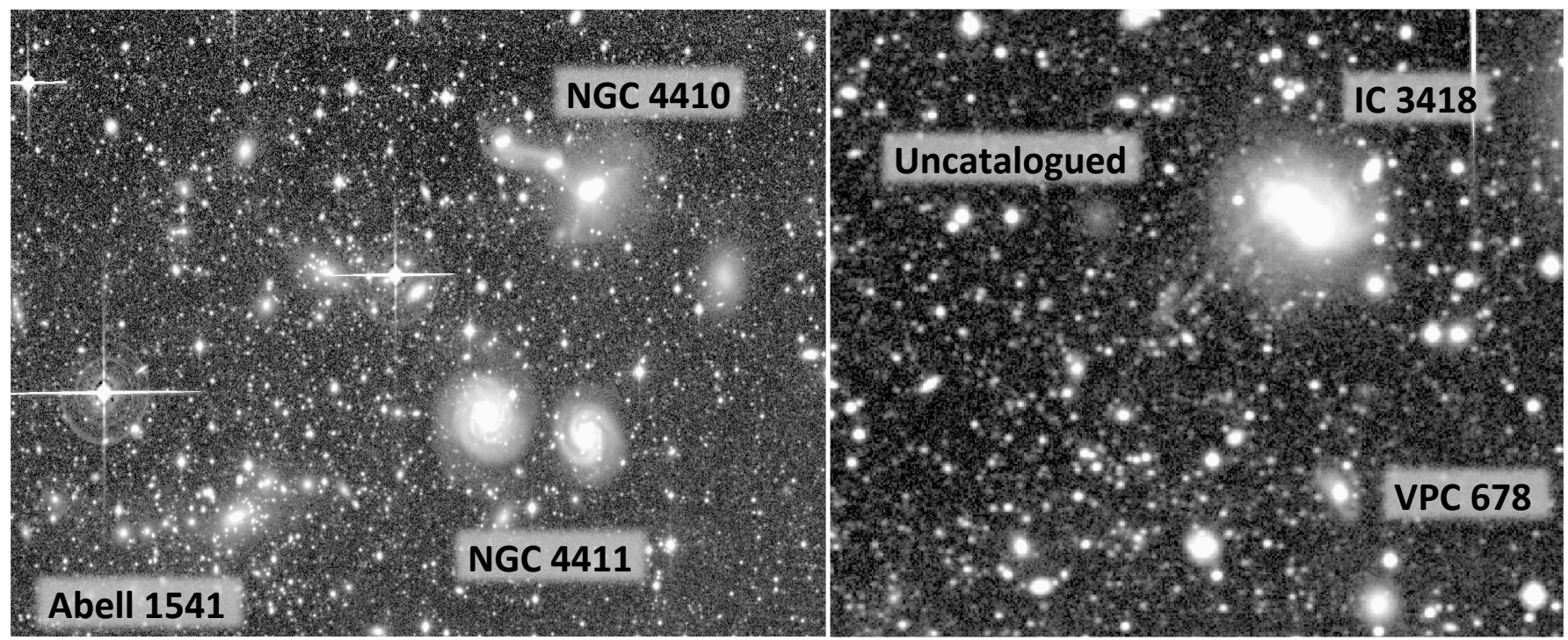

Figure 4. Two fields showing the variety of diffuse features we see in the imaging data. Left: $35^{\prime} \times 24^{\prime}$ cutout of the V mosaic showing diffuse light in the distant cluster Abell $1541(z=0.09)$, the tidal plumes associated with the background galaxy group around NGC 4410 $\left(c z=7254 \mathrm{~km} \mathrm{~s}^{-1}\right)$, and the faint outer spiral arms of the Virgo galaxy pair NGC 4411. Right: $12^{\prime} \times 10^{\prime}$ cutout of a field near the ram presssure stripped galaxy IC 3418 (Hester et al. 2010, Kenney et al. 2014), showing the galaxy's diffuse outer regions and its star forming knots extending to the southeast, an uncatalogued low surface brightness galaxy at unknown redshift, and extensive tidal debris surrounding the background galaxy VPC $678(z=0.18)$. These fields represent only $\sim 1 \%$ of the full V mosaic; similar structures abound throughout the other $99 \%$ of the survey area.

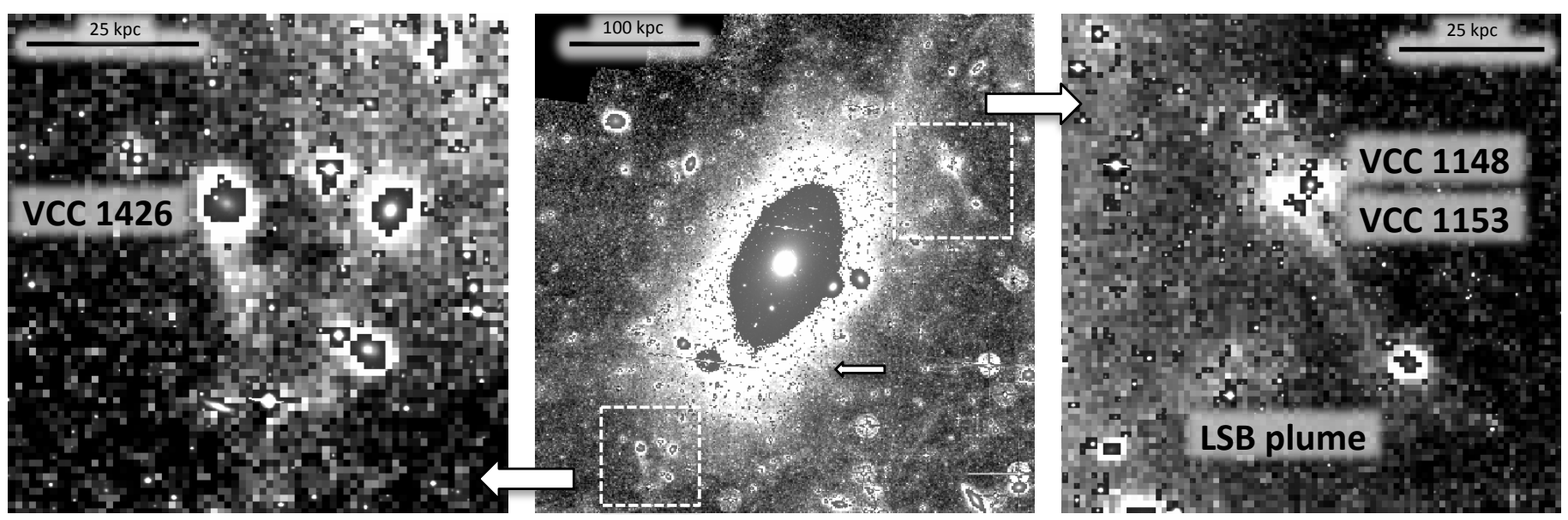

Figure 5. Streams around M87. The $1.3^{\circ} \times 1.3^{\circ}$ center image shows M 87 itself, with two subfields highlighted. The left panel shows a $0.25^{\circ} \times 0.25^{\circ}$ subfield around VCC 1426 , while the right panel shows a $0.33^{\circ} \times 0.33^{\circ}$ subfield around VCC 1153 . In all three panels, at high surface brightnesses $\left(\mu_{V}<26\right.$ mag arcsec $\left.{ }^{-2}\right)$ the imaging is shown at full $1.45 \operatorname{arcsec~pixel~}^{-1}$, while at lower surface brightnesses we show the $9 \times 9$ median binned image using a remapped intensity scale to highlight faint structures. In the center figure, the arrow points to a small linear stream projecting out from M87's halo. See text for details.

Jordán et al. 2004; De Lucia \& Blaizot 2007; Guo et al. 2011; Tovmassian \& Andernach 2012).

Indeed, signatures of more massive mergers may be lurking within M87's halo. Kinematic studies of M87's globular clusters (Romanowsky et al. 2012) and $\mathrm{PNe}$ (Longobardi et al. 2015a) suggest the recent accretion of one or more systems with total mass $\sim$ a few $\times 10^{9}$ to $10^{10} \mathrm{M}_{\odot}$. Similarly, M87's isophotal structure hints at past accretion events as well. While the large extent of the galaxy's diffuse halo was first noted by Arp \& Bertola (1969), Carter \& Dixon (1978) were the first to point out the asymmetric nature of the outer isophotes. Weil, Bland-Hawthorne, \& Malin (1997) later used deep photographic imaging to trace out a diffuse fan of material emanating from the SE of the galaxy, as well as a smaller "cap" of material to the NW, indicative of a recent accretion event. In an earlier analysis of our Virgo imaging data, we showed a broad but faint plume of material north of M87 (Rudick et al. 2010), while Longobardi et al. (2015a) used the updated imaging described here to argue that the NW isophotal structure (Weil et al. 's "cap," referred to in Longobardi et al. 2015a as the "crown") at $800^{\prime \prime}-1200^{\prime \prime}$ trace the discrete accretion event implied by the PNe kinematics.

Figure 6a shows our $B$ imaging around M87, color coded by surface brightness, where we see large scale systematic changes with radius in M87's isophotal structure. The mild boxiness of the inner regions of the galaxy transitions to a more disky morphology at $10^{\prime}$, where the surface brightnesses has dropped to $\mu_{B} \sim 27$ (the light green 

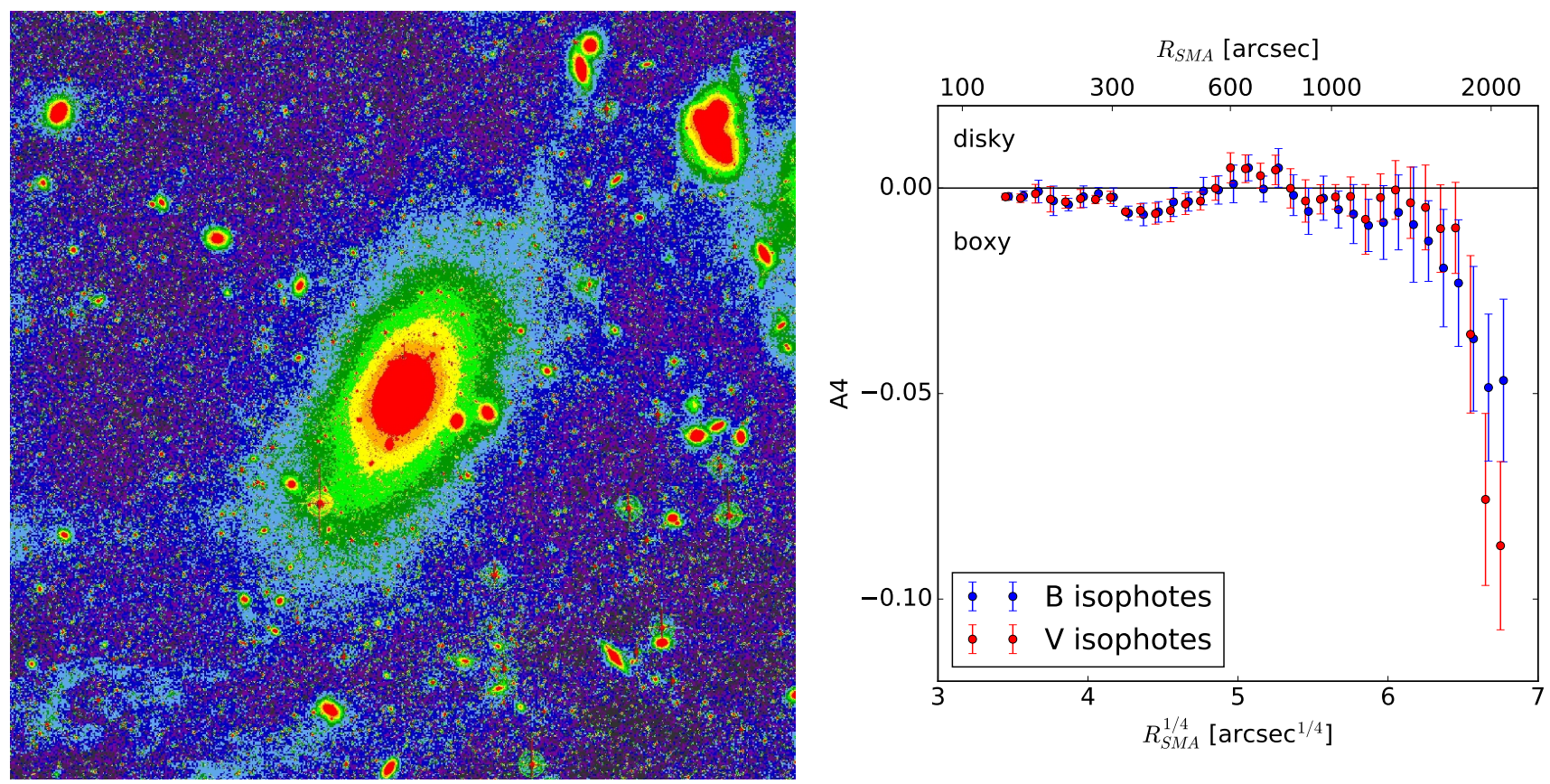

Figure 6. Left: Deep $B$ band image of M87, $2^{\circ}\left(550 \mathrm{kpc}\right.$ ) on a side, color-coded by surface brightness: $\mu_{B}<25$ (red), $25<\mu_{B}<25.75$ (orange), $25.75<\mu_{B}<26.5$ (yellow), $26.5<\mu_{B}<27.25$ (light green), $27.25<\mu_{B}<27.75$ (dark green), $27.75<\mu_{B}<28.5$ (light blue), $\mu_{B}>28.5$ (dark blue). Right: Amplitude of the $\cos (4 \theta)$ term in the isophotal analysis, plotted as a function of semi-major axis distance.

isophotes of Figure 6a. At larger radius, the isophotes revert back to become extremely boxy; the large NW extent of the isophotes, traced beyond the cap/crown structure to nearly $1800^{\prime \prime}(140 \mathrm{kpc})$ along the major axis balances the fan of material to the SE, and gives rise to the overall symmetric shape of the galaxy's boxy outer isophotes at low surface brightness $\left(\mu_{B}=27.5\right.$; the light blue isophotes in Figure 6a. Here in the outer halo, the boxiness of the isophotes is echoed in the boxiness of the spatial distribution of M87's globular cluster population as well (Durrell et al. 2014). While we note the presence of patchy galactic cirrus in the extreme southeast portion of the field, an examination of the Herschel $250 \mu$ m map (Figure 3) shows no evidence that this contamination extends into the outer isophotes of M87's halo studied here.

We quantify the boxiness of the isophotes by using IRAF's ellipse task (Jedrzejewski 1987, Busko 1996) to fit elliptical isophotes to M87 and measure the harmonic deviations of the isophotes from pure ellipses. We show the amplitude of the $\cos (4 \theta)$ harmonic as a function of semi-major axis radius. in Figure 6b. The sign of this A4 coefficient measures isophotal shape, with positive values indicating diskiness and negative values indicating boxiness. To assess the uncertainty in the A4 terms, we ran 100 iterations of the ellipse algorithm, varying task parameters such as the initial fitting radius, radial step size, and input photometric parameters; errorbars in Figure 6 6 reflect the $1 \sigma$ scatter in the extracted A4 terms. In addition, we measure the isophotal shape independently on each of the $B$ and $V$ images, obtaining consistent results between the two images, and note that the V-band surface brightness, ellipticity, and position angles, while not shown, are consistent with our earlier analysis in $\mathrm{J}+10$.

This quantitative analysis confirms the visual impression of boxiness evident in the image. Between $r^{1 / 4}=4-5$ $\left(4^{\prime}-10^{\prime}\right.$, or $\left.\sim 20-50 \mathrm{kpc}\right)$ the A4 terms are slightly negative, indicative of a somewhat boxy shape, then switch sign around $50 \mathrm{kpc}$, indicating diskiness. This behavior is also clearly seen in the M87 surface photometry of Kormendy et al. (2009). The switch to disky isophotes can be seen morphologically in Figure 6a as traced by the light green isophotes at $\mu_{B} \sim 27$. At even larger radius, $r^{1 / 4}>6$, or $r>100 \mathrm{kpc}$, the A4 terms become strongly negative, reflecting the extreme boxiness of the outermost isophotes. Such extreme boxiness is atypical of the inner regions of bright ellipticals, which rarely show A4 terms more negative than -0.01 (Bender 1989, Emsellem et al. 2011).

The connection between isophotal shape and assembly history of the galaxy is complex. Numerical simulations of merging galaxies generally show that boxiness is associated with major mergers (particularly dry mergers), while unequal mass minor mergers, or mergers with significant gaseous dissipation, tend to leave diskier remnants (e.g., Khochfar \& Burkert 2005, Naab et al. 2006). However, simple single merger models may not be applicable to M87 - as Virgo's central BCG it has likely undergone a complex series of mergers with varying mass ratios, orbital energies, and angular momenta. Simulations indicate that the growth of cluster BCGs is marked by multiple accretions, with as much as $50 \%$ of the BCG mass being accreted since $z=0.5$ in the form of intermediate mass companions (with masses $>10^{10} \mathrm{M}_{\odot}$; de Lucia \& Blaizot 2007, Cooper et al. 2015). M87's extended halo is likely therefore made up of an ensemble of material from past accretion events that may not be fully relaxed, and the various structures seen at low surface brightness (the NW streams, SE fan, N plume, and NW cap/crown) may conspire to yield M87's varying A4 profile and extremely boxy outer isophotes shown in Figure 6 In this sense, M87's halo morphology at low surface brightness is quite reminiscent of simulated BCGs of 
Cooper et al. (2015), whose boxy halo morphology arises at least in part from a superposition of variety of tidal debris structures from many past accretion events.

\subsection{The M86/M84 Field}

Another area of high projected galaxy density in Virgo is the region containing the massive ellipticals M86 and M84, although the two ellipticals themselves may be well separated along the line of sight (e.g., Mei et al. 2007). The presence of an extensive, diffuse ICL component in this region is indicated by the large number of intracluster PNe found in the field (Arnaboldi et al. 1996, Okamura et al. 2002), as well as by the discovery of an intracluster Type Ia supernova (Smith 1981). As shown in $\mathrm{J}+10$, the halos of both M86 and M84 contain a number of small streams suggestive of tidal stripping of dwarf galaxies orbiting the massive ellipticals. component in this region as well. Here we concentrate on the features seen around other, smaller galaxies in this field. Figure 7 shows the region just south of M86 and M84, where many of the galaxies show diffuse features. While it is tempting to interpret these features as signs of interaction with one another, the galaxies in this field show a wide range of velocities, from $88 \mathrm{~km} \mathrm{~s}^{-1}$ (NGC 4413; Rines \& Geller 2008 ) to $2513 \mathrm{~km} \mathrm{~s}^{-1}$ (NGC 4388), and thus are not likely involved in close and slow encounters with one another that might lead to the strongest tidal response.

The edge on disk galaxy NGC $4425\left(1899 \mathrm{~km} \mathrm{~s}^{-1}\right)$ shows a bright plume extending $3.5^{\prime}$ to its south, originally identified in photographic imaging by Malin (1994). The plume is brightest at its southernmost tip, where it has a peak surface brightness of $\mu_{V}=26.0$ and color of $B-V=0.76$. While tidal tails often show condensations of stars and gas (e.g., Hibbard et al. 1994, Duc et al. 2000, Weilbacher et al. 2000, Lelli et al. 2015), in this case the tip of the plume has a fairly constant surface brightness with no sign of any central condensation. This morphology is somewhat suggestive of edge-brightening, where an optically thin and curved tidal tail is observed edge on. At the point where the tail curves back in projection along the line of sight the observational path length through the tail is maximized and the projected surface brightness thus increases. The position angle of the tail is offset by $50^{\circ}$ from the plane of the galaxy's disk, arguing that the tail formed during an interaction that occurred out of the galaxy's disk plane.

The other edge-on spiral galaxy in the field, NGC 4388 $\left(2513 \mathrm{~km} \mathrm{~s}^{-1}\right)$, also shows low surface brightness features extending from both sides of its major axis. The system shows ionized emission extending $7^{\prime}$ to the northeast (Yoshida et al. 2002), as well as an even more extended HI tail stretching nearly $30^{\prime}$ along the same direction, likely due to ram pressure stripping in the cluster environment (Oosterloo \& van Gorkom 2005). At the base of the HI tail, near the region of ionized emission, we also detect diffuse light at $\mu_{V}=27.5$ with a broadband color ( $B-V=0.66)$, somewhat bluer than the tidal features we see elsewhere in Virgo. On the other side of the disk, the western plume is even bluer, with colors ranging from $B-V=0.5-0.6$, suggestive of young stars being stripped from the galaxy's disk, or perhaps collisionally-induced star formation in the tidal feature. Given the intracluster HII region projected $3.5^{\prime}(17 \mathrm{kpc})$ to the north of the galaxy (Gerhard et al. 2002) as well, the complex struc- ture around NGC 4388 is a good example of how tidal stripping and ram pressure stripping can work in tandem to deposit young stars into the intracluster environment.

The outer isophotes of the inclined spiral NGC 4413 (88 $\mathrm{km} \mathrm{s}^{-1}$, Rines \& Geller; the galaxy is also cataloged as NGC 4407) show more complex structure. There is a diffuse stream of starlight arcing west towards NGC 4388, and another extending southeast towards IC 3363. Both streams are quite red $(B-V=0.9-1.0)$, and are morphologically similar to the two armed "bridge/tail" morphology of spiral galaxy encounters (e.g., Toomre \& Toomre 1972). While the colors of the streams are significantly redder than the interior of NGC $4413(B-V=0.62)$, the galaxy's disk shows a redward color gradient - at larger radius the colors are $B-V=0.7-0.8$, and the outermost parts of many spiral disks show even redder colors (Bakos et al. 2008, Zheng et al. 2015, Watkins et al. 2016). Thus the colors are consistent with stripping of the disk outskirts, although the identity of the perturbing companion remains ambiguous. The mismatch in velocity with both NGC 4388 and IC 3363 means that even if these galaxies are in close proximity to NGC 4413, any interaction would be fast and less able to trigger a strong tidal response. On the other hand, NGC 4413's velocity suggests membership in the M86 subgroup, where an interaction with the massive elliptical might be responsible for the observed tidal debris. However, without accurate distances, it is impossible to determine this with any certainty.

The three small galaxies IC $3363\left(851 \mathrm{~km} \mathrm{~s}^{-1}\right)$, VCC 962 (velocity unknown), and IC 3349 (1411 $\mathrm{km} \mathrm{s}^{-1}$ ) are all embedded in a diffuse halo of light which trails off in intensity to the southwest. This halo has a surface brightness of $\mu_{V}=27.5$ and color $B-V=0.9$, and IC 3363 itself shows a detached, diffuse region of higher surface brightness $\left(\mu_{V} \sim 26, B-V=0.75\right)$ directly to its north. The large $\left(560 \mathrm{~km} \mathrm{~s}^{-1}\right)$ velocity difference between IC 3363 and IC 3349 makes it unlikely that this trio of galaxies is a true physical group, and the diffuse halo surrounding them may simply be material seen in projection.

Also extending to the SE along the major axis of M86 is a broad shelf of light which does not trace the elliptical isophotes of the giant elliptical. This feature, not originally identified in the analysis of $\mathrm{J}+10$, has a surface brightness of $\mu_{V}=27.3$ and $B-V$ color of 0.85 . Given its rather large size $\left(\sim 40 \operatorname{arcmin}^{2}\right)$ the total luminosity in this feature is significant: $L=3.5 \times 10^{8} \mathrm{~L} \odot$. Unlike the majority of the streams J+10 identified in M86's halo, which are typically smaller and lower in luminosity than this "SE shelf," this feature is more similar to the broad accretion structures seen in M49 and M87, and may be tracing an older and more massive accretion event in M86's past.

\subsection{The M49 Field}

The giant elliptical M49 (981 $\mathrm{km} \mathrm{s}^{-1}$; Cappellari et al. 2011) hosts an intricate system of debris shells, described in detail in our earlier papers ( J+10, Mihos et al. 2013a), and seen also in deep imaging by the NGVS and VEGAS teams (Arrigoni Battaia et al. 2012 and Capaccioli et al. 2015, respectively). Figure 8 shows the more extended field around M49, where the NW and SE shells can be 


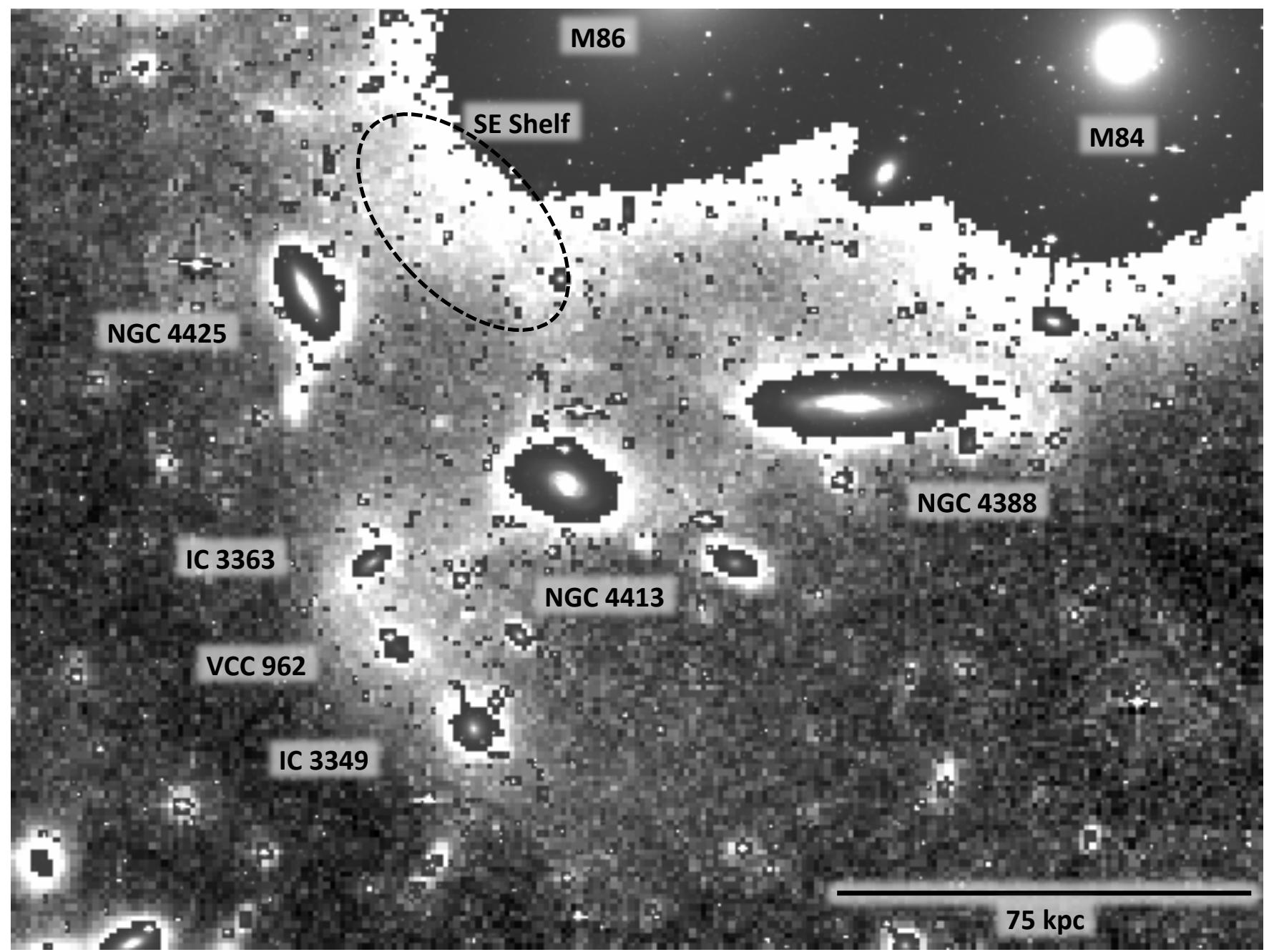

Figure 7. Deep V image of the M86/M84 field. The image covers a field of view $48^{\prime} \times 36^{\prime}$ in size, with north up and east to the left. Galaxies discussed in the text are marked, as is the SE Shelf.

seen in M49's outer halo.

At lower surface brightness we find a stream of extremely diffuse light in the field northeast of M49, along a line roughly connecting to the spiral galaxy NGC 4519 $\left(1241 \mathrm{~km} \mathrm{~s}^{-1}\right)$. The stream is $\sim 15^{\prime}(70 \mathrm{kpc})$ long, with a surface brightness of $\mu_{V}=28.5$. There is no corresponding feature in the HeVICS $250 \mu$ map (Figure 33, arguing that it is not scattered light from galactic dust. The alignment of the feature suggests instead that it may be a stream formed from a past encounter between NGC 4519 and M49, although the large projected separation between the galaxies $\left(68^{\prime}, 325 \mathrm{kpc}\right)$ would place any close passage at least 1 Gyr in the past. NGC 4519 itself is also distorted, and interacting with its close companion NGC 4519A (1434 $\mathrm{km} \mathrm{s}^{-1}$; Binggeli et al. 1985) just to the northwest; if this is a long lived interaction, it could have acted to strip material from NGC 5419 that was then subsequently drawn out into the longer diffuse stream by the tidal interaction with M49.

We also find a rather amorphous patch of diffuse light $41^{\prime}(190 \mathrm{kpc})$ to the west of M49. It lies at a position $(\alpha, \delta)=(12: 27: 05,+8: 00: 38)$, midway between the galaxies NGC 4434 and NGC 4416 (which themselves are separated by $19^{\prime} / 90 \mathrm{kpc}$ ), but we so no evidence of diffuse light connecting the patch with either of those galaxies, or with M49 itself. The patch covers $\sim 9$ square arcminutes $\left(200 \mathrm{kpc}^{2}\right)$, and has a roughly uniform surface brightness of $\mu_{V}=27.5$ and $B-V$ color of 0.9 . With an irregular structure and no central concentration, it is unlike known ultradiffuse galaxies in Virgo (Mihos et al. 2015, Beasley et al. 2016) and Coma (van Dokkum et al. 2015, Koda et al. 2015), and while it could be a localized patch of Galactic cirrus, we see no corresponding farinfrared emission in the $250 \mu$ HeVICs map. Its origin thus remains unclear.

Aside from these two features and the shell system surrounding M49, we find no extended diffuse light or small tidal streams in the field brighter than $\mu_{V}=29.0$. Deeper inside M49's halo itself, the only ongoing accretion event involves the stripping of the dwarf irregular VCC 1249 (McNamara et al. 1994; Lee et al. 2000; Arrigoni Battaia et al. 2012). This is in marked contrast to the high density fields around M87 and M86/M84 and suggests that the current rate of ICL production around M49 is lower than that in the cluster core, as might be expected if ICL production traced either galaxy density or cluster potential well. Nonetheless, while there are few ongoing interactions around M49 today, the accretion rate onto its halo may be similar to that onto M87. Outside of the cluster core, where it is not subject to repeated bom- 
bardment by infalling satellites, tidal debris can survive on Gyr timescales. If we add the observed luminosity of M49's shell system $\left(\sim 7.3 \times 10^{8} \mathrm{~L}_{\odot, V} ; \mathrm{J}+10\right)$ and the VCC 1249 streams 7 (roughly another $10^{8} \mathrm{~L}_{\odot, V}$ ), factoring in the Gyr survival timescale implies a rough accretion rate on the order of $\sim 1 \mathrm{~L}_{\odot, V} \mathrm{yr}^{-1}$. The fact that M49 is similar to M87 in both total luminosity and current low mass accretion rate again argues that the halo growth of massive ellipticals is dominated by major or past accretion history, and that present day satellite accretions are only a minor source of additional luminosity.

Meanwhile, M49 itself is thought to be falling into the Virgo Cluster from the south, as evidenced by the X-ray bow shock to the north of M49 (Irwin \& Sarazin 1996, Kraft et al. 2011). When the galaxy enters the denser environment of the Virgo Cluster core, its delicate system of shells will likely be disrupted due to encounters with other galaxies and the cluster potential itself, adding to Virgo's growing ICL component.

\subsection{The NGC 4365 Field}

The giant elliptical NGC 4365 (1243 km s ${ }^{-1}$; Cappellari et al. 2011) lies in the lower right (southwest) corner of our imaging fields (Figures 1 and 2), 5.3 $\mathrm{SSW}$ of M87 and $1.5^{\circ} \mathrm{WSW}$ of M49. It is located $\sim 6 \mathrm{Mpc}$ behind the Virgo Cluster (Mei et al. 2007) ${ }^{8}$ and is the dominant galaxy of the Virgo $W^{\prime}$ group (de Vaucouleurs 1961, Binggeli et al. 1985; Mei et al. 2007), surrounded by a population of lower luminosity galaxies (see Figure 9a) within $\Delta v= \pm 350 \mathrm{~km} \mathrm{~s}^{-1}$ and a projected distance of $1^{\circ}(400 \mathrm{kpc})$. NGC 4365 itself has a kinematically distinct core (Bender \& Surma 1992), along with evidence for multiple populations of globular clusters (Blom et al. $2012 b)$. An early release of our Schmidt imaging data, shown in Bogdán et al. (2012), revealed an extended tidal tail extending SW from NGC 4365. A number of companion galaxies are projected on or near the tail, most notably the S0 galaxy NGC $4342\left(761 \mathrm{~km} \mathrm{~s}^{-1}\right.$, Cappellari et al. 2011). While Bogdán et al. argued the tail was unlikely to have been stripped from NGC 4342, due to the high dark halo mass implied by the galaxy's Xray luminosity, subsequent observations by Blom et al. (2012a, 2014) of globular clusters in the stream showed a convincing kinematic match between the tidal tail and NGC 4342.

Figure 9b shows a deep stretch of our $B$ band image, after masking of bright sources. The long tidal tail can easily be seen projecting $35^{\prime}$ to the southwest, with both NGC $4341\left(922 \mathrm{~km} \mathrm{~s}^{-1}\right)$ and NGC $4342 \mathrm{em}-$ bedded within. The brightest part of the tail, southwest of NGC 4342, reaches a peak surface brightness of $\mu_{V}=27.25$ before making a $90^{\circ}$ dogleg turn at its southwest limit, becoming more diffuse and extending another $14^{\prime}$ to the northwest. The tail has a $B-V$ color of $\sim$ 0.75-0.85, somewhat bluer than the integrated color of either NGC 4365 or NGC $4342(B-V=0.96$ and 0.95 , respectively, RC3). However, both galaxies show a marked

\footnotetext{
7 If we assume that VCC 1249 will be completely disrupted, adding in its entire $\mathrm{V}$ luminosity rather than just that in its tidal streams increases our inferred accretion rate by only $\sim 40 \%$.

8 For the discussion of physical sizes and luminosities for features around NGC 4365, we adopt a distance of $23 \mathrm{Mpc}(m-M=31.8$; Mei et al. 2007) to the system, for which $1^{\prime \prime}=112 \mathrm{pc}$.
}

color gradient: at $40^{\prime \prime}, \mathrm{NGC} 4342$ has a mean color of $B-V=0.8$, as do NGC 4365's outer isophotes at $500^{\prime \prime}$ (Figure 10). While the color of the tail is consistent with tidal stripping from the outer regions of either galaxy, morphological arguments favor a scenario involving stripping from NGC 4342. The outer isophotes of NGC 4342 are elongated along the direction of the tidal tail, and the thickness of the tail $\left(5^{\prime}\right)$ is similar to the isophotal size of NGC 4342. Also, stripping of the outer regions of a dynamically hot, massive elliptical like NGC 4365 would likely lead to a more diffuse plume of starlight rather than the long, thin tail seen in Figure 9. Given its size and surface brightness, we estimate a total magnitude for the tail of $m_{V} \sim 12.5$, comparable to that of NGC 4342 itself (RC3). As such, the interaction between the galaxies has led to a significant loss of stellar material from NGC 4342, as also traced by the kinematics of the globular clusters in the tidal tail (Blom et al. 2012a, 2014). How the galaxy could have been so severely stripped of its stellar material, while retaining its massive dark halo (as traced by its X-ray emission; Bogdán et al. 2012) remains unclear, although as suggested by Blom et al. , resonant stripping of NGC 4342's rotating disk may preferentially strip the galaxy's stars while leaving its dark halo relatively intact (D'onghia et al. 2009).

Also visible in Figure 9 is a curved stream of diffuse starlight to the northeast of NGC 4365, coincident with the companion galaxy VCC $755\left(1241 \mathrm{~km} \mathrm{~s}^{-1}\right)$. The stream is relatively thin $\left(2^{\prime}\right)$ and most clearly delineated over a $12.5^{\prime}$ segment where it emerges from the northeast of VCC 755 and is then lost in the halo of a nearby bright star. Here the stream has a surface brightness of $\mu_{V}=28.0, B-V$ color of 0.8 , and total magnitude $m_{V}=15.6$. To the southwest of VCC 755 we see more diffuse light apparently connecting the galaxy to NGC 4365, but this component is broader and higher in surface brightness $\left(\mu_{V}=27.0\right)$ making it unclear whether this is a continuation of the northeast stream or an independent structure embedded in the halo of NGC 4365. VCC 755 itself is a low surface brightness dwarf $\left(\mu_{V}=22.8, m_{V}=16.2, B-V=0.85\right)$; the similarity in color between the galaxy and the stream supports the idea that the stream originates from tidal stripping of VCC 755, which must be significant given that the stream contains significantly more starlight than the galaxy itself.

Finally, we identify an asymmetric plume of light in the southern isophotes of NGC 4365's halo. This feature is broad and diffuse $\left(\mu_{V}=27.5\right)$, and is not obviously associated with any nearby companion galaxy. It is similar in color to the outskirts of NGC $4365(B-V=0.85)$ and may trace an older accretion event that has deposited starlight into the galaxy's halo.

To better understand the connection between these various diffuse features around NGC 4365, we also fit and subtract a smooth elliptical isophotal model for NGC 4365, using the procedure described in $\mathrm{J}+10$. Briefly, we mask bright stars, neighboring galaxies, and faint discrete sources in the field using IRAF's objmask task, then run its ellipse task to fit an elliptical model to NGC 4365's light profile. This model profile (see Figure 10 is then subtracted from the original image, and the residual image is then median binned in $9 \times 9$ pixel blocks to bring out the faintest features, shown in 9 . 


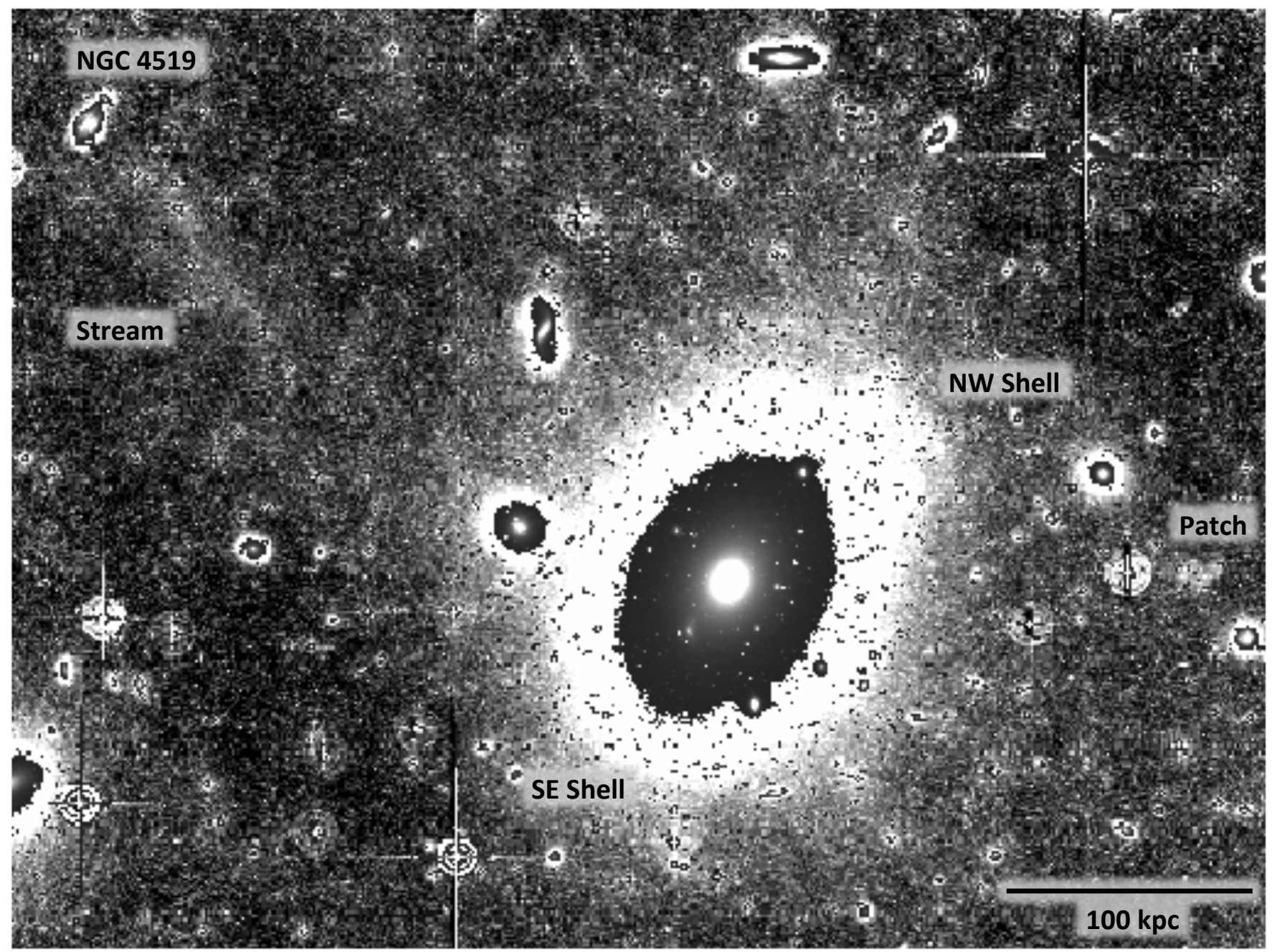

Figure 8. Deep V image of the M49 field covering a field of view $1.8^{\circ} \times 1.4^{\circ}$ in size. North is up and east is to the left, and features discussed in the text are marked.

All the structures discussed in this section are visible in the residual map, demonstrating that they are discrete structures distinct from the smooth halo of NGC 4365. However, we see no evidence of continuity between the SW tail and either the NE stream or S plume, arguing that these are independent structures tracing different accretion and interaction events around NGC 4365.

The field around NGC 4365 thus illustrates an important first step in generating diffuse intracluster light: the "pre-processing" of galaxies via close encounters in the group environment. The total amount of diffuse light and complex structure in the tidal debris is reflective of the multiple close interactions that take place in dense group environments (e.g., Weil \& Hernquist 1996, Rudick et al. 2006, Durbala et al. 2008). In observed compact groups, the fraction of diffuse light is thought to be very high, $10 \%$ or more (Da Rocha \& Mendes de Oliveira 2005, Da Rocha et al. 2008). While the diffuse light fraction we observe around NGC 4365 is lower than this $(\sim 3 \%)$, it is higher than we observe in most other massive Virgo ellipticals (typically $\sim 0.5 \%$ or less; J+10). With a single massive elliptical and a swarm of smaller companions, the NGC 4365 group is likely more evolved than typical compact groups which have multiple comparably massive galaxies, and much of the material stripped early in the group's history has likely now been incorporated into NGC 4365's luminous halo. As NGC 4365 and its companions eventually fall into the core of Virgo, much of this diffuse material will be stripped away from the group and dispersed throughout the cluster core (e.g., Rudick et al. 2006), further feeding Virgo's diffuse intracluster light.

\subsection{VLSB-D: A new ultradiffuse galaxy}

Aside from the streams and shells of tidal debris we find in Virgo, our deep imaging data also allows us to search for extremely low surface brightness galaxies that may populate the cluster. Of particular interest here is finding Virgo analogues to the population of large and faint "ultradiffuse galaxies" (UDGs) that have recently been identified in deep imaging of the Coma cluster (van Dokkum et al. 2015, Koda et al. 2015). In our imaging, we see many examples of small LSB objects (see, for example, Figure 4), but without distance information it is hard to place them uniquely in the Virgo cluster. Furthermore, even if they are at the Virgo distance, their small sizes means they are not truly UDGs (which have effective radii $r_{e}>1 \mathrm{kpc}$, or $12.5^{\prime \prime}$ at Virgo), but rather part of the well-known population of LSB dwarfs that teem inside galaxy clusters (e.g., Impey et al. 1988; Fer- 

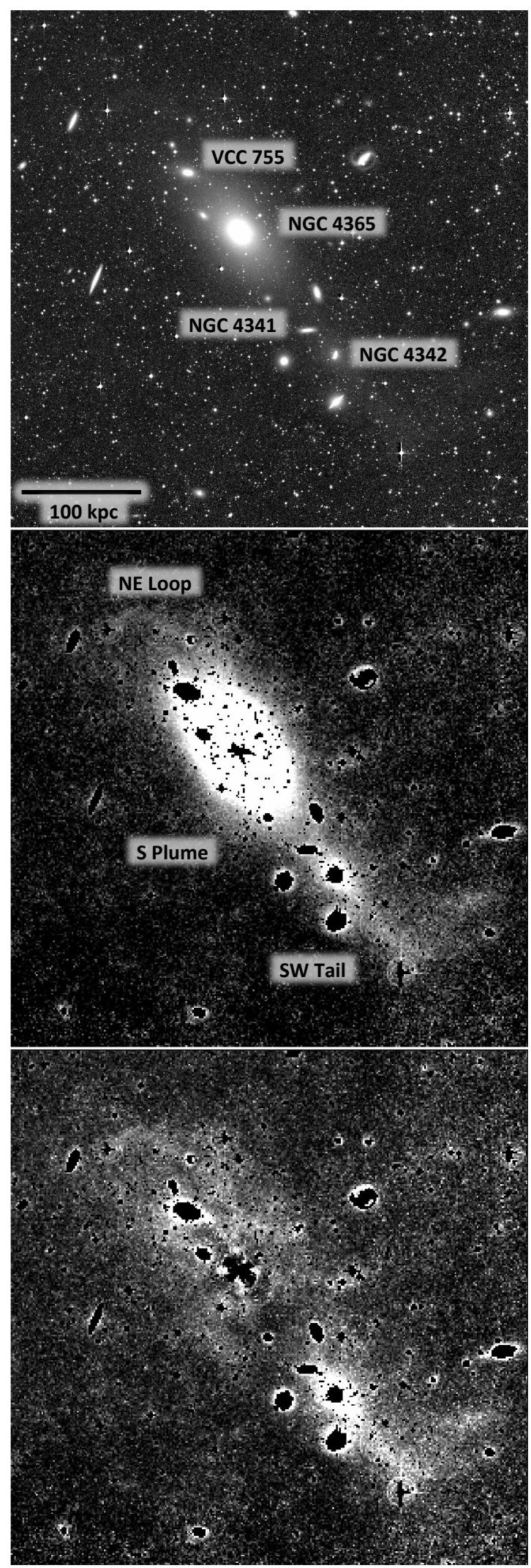

Figure 9. $B$ band imaging of NGC 4365. Each image spans $1.1^{\circ}$ on a side, with north at the top and east to the left. Top: Full resolution image. Middle: Deep image, after masking discrete structures and median smoothing to $9 \times 9$ pixel $\left(13^{\prime \prime} \times 13^{\prime \prime}\right)$ resolution. Bottom: Masked and smoothed image after subtraction of an elliptical isophotal model for NGC 4365. North is up, east to the left. See text for details.

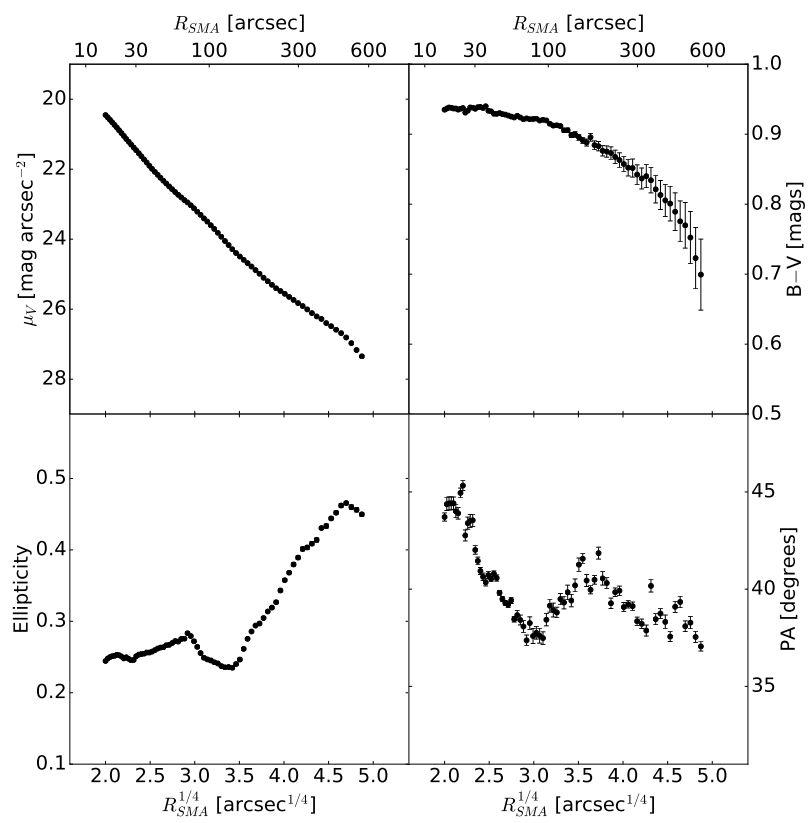

Figure 10. Photometric profile of NGC 4365, showing the V surface brightness, $B-V$ color, and isophotal ellipticity and position angle. Errorbars in surface brightness and color are based on a residual sky uncertainty of $0.5 \mathrm{ADU}$, while errorbars on ellipticity and position angle are calculated as in Busko (1996). In surface brightness and ellipticity, the errobars are smaller than the point size.

rarese et al. 2016). However, we also find a handful of very large $\left(r_{e}>30^{\prime \prime}\right)$ and diffuse $\left(\langle\mu\rangle_{e, B}>28\right)$ systems in our imaging; our earlier paper (Mihos et al. 2015) discusses three such objects found in the deep V-band mosaic. Here we add one more UDG to that list: an object located on the B-band mosaic only, of large angular size and extremely low surface brightness.

Figure 11 shows the imaging and extracted surface brightness profile for this new object. Located at $(\alpha, \delta)=(12: 24: 42.1,+13: 31: 02)$, it is projected $\sim 38^{\prime}(180$ kpc) north of the bright elliptical M84. The system is extremely elongated in the $\mathrm{N}-\mathrm{S}$ direction, and has a compact source at its center. While we have no color information from our data (as it falls only in the $B$-band footprint), the compact source is detected in SDSS with $g=20.72$ and $g-r=0.58$, a color similar to luminous Virgo globular clusters (e.g., Durrell et al. 2014) and ultra-compact dwarf galaxies (UCDs; Liu et al. 2015). Excluding the central source, the object has a central surface brightness of $\mu_{B}=27.1$ and a total magnitude of $m_{B}=16.3$ within a $150^{\prime \prime}(12 \mathrm{kpc})$ extent. However, there is additional light beyond this radius, suggestive of a tidal stream to the north and perhaps also to the south, although the presence of bright stars and other sources in this direction makes it difficult to measure the profile. The surface brightness profile shows an exponential form; a Sersic fit (shown in Figure 11) is virtually indistinguishable from an exponential fit, and yields a Sersic index $n=0.97 \pm 0.14$, effective radius of $r_{e}=166 \pm 25^{\prime \prime}(13.3 \pm 2$ $\mathrm{kpc})$, mean surface brightness $\langle\mu\rangle_{e, B}=28.2 \pm 0.2$, and total magnitude $m_{B, t o t}=15.5 \pm 0.4$. However, given the object's extreme flattening and irregular, extended outer isophotes, it is likely not a system in dynamical equilib- 
rium, making the interpretation of the structural properties derived from the fit rather questionable. Nonetheless, the extremely large size and low surface brightness of the object makes it one of the most extreme UDGs yet discovered.

The properties of this object are similar to the Virgo UDG VLSB-A (Mihos et al. 2015) - a large, low surface brightness galaxy with extended tidal debris and a compact source at its center. In both cases, we are likely seeing the tidal destruction of a nucleated LSB galaxy, plausibly leading to the formation of a new Virgo UCD (e.g., Bekki et al. 2003; Pfeffer \& Baumgardt 2013). The fact that two of the four very large UDGs discovered in our deep imaging data appear in this short-lived phase suggests that a significant fraction of cluster UDGs - at least in cluster cores, where our imaging is targeted may be actively being stripped, and that this mechanism is a common way of producing UCDs. However many UDGs show little sign of tidal deformation (van Dokkum et al. 2015, Mihos et al. 2015) and at least some also appear to possess robust globular cluster systems (Mihos et al. 2015, Beasley et al. 2016, Peng \& Lim 2016), suggesting they are rather robust against tidal stripping. Given the loose definition of UDGs, and the wide range of properties they display, it would not be surprising if, as a class, they were comprised of galaxies drawn from a variety of of evolutionary histories.

\section{DIFFUSE LIGHT AND THE DYNAMICAL EVOLUTION} OF THE VIRGO CLUSTER

A variety of dynamical modeling studies have shown that the build up of intracluster light, like the growth of structure in the universe in general, is a hierarchical process. As smaller systems interact and merge to form larger galaxies, stars are stripped into the outskirts of their host galaxies. When galaxies assemble into groups, this stripping accelerates, as the loosely-bound material in the galaxies' outskirts is shorn away during repeated encounters in the group environment. Eventually, as the groups fall into a more massive cluster environment, this intragroup light is easily stripped away by interactions with massive central galaxies and the cluster potential itself (Rudick et al. 2006, Purcell et al. 2007, Contini et al. 2014), and additional stripping is mediated both by the merging of massive galaxies in the cluster core (Murante et al. 2007, Martel et al. 2012) and by high speed encounters between galaxies orbiting within the cluster environment (Moore et al. 1996). In this way, both the amount and distribution of intracluster light are intimately linked to the dynamical evolution of the cluster (Rudick et al. 2006, 2011). Low density environments such as poor clusters and loose groups would have less diffuse light, while dense, highly evolved groups and clusters should possess the largest amounts of intracluster light (e.g., Rudick et al. 2006, Sommer-Larsen 2006, Contini et al. 2014). However, at best the ICL content of a cluster scales only weakly with cluster mass; simulated clusters show significant scatter in their ICL fractions at fixed mass, and the driving influence in ICL production seems to be environmental density and accretion history rather than total mass (e.g., Conroy et al. 2007, Rudick et al. 2011, Contini et al. 2014).

With this backdrop, it is useful to examine Virgo's diffuse light as a tracer of the cluster's evolutionary state, and assess the observational link between cluster dynamics and the generation of ICL. A cluster of moderate virial mass $\left(M_{v i r} \approx 1-4 \times 10^{14} \mathrm{M}_{\odot} ;\right.$ McLaughlin 1999 , Urban et al. 2011), Virgo shows signs of ongoing assembly, with ample evidence for substructure on both large and small scales in the spatial distribution, kinematics, and morphologies of its galaxy populations (e.g., Binggeli et al. 1985, 1987, 1993; Gavazzi et al. 1999; Mei et al. 2007, Kim et al. 2014) as well as in tracer populations such as globular clusters (e.g., Romanowsky et al. 2012, Durrell et al. 2014) and planetary nebulae (Arnaboldi et al. 2004, Doherty et al. 2009, Longobardi et al. 2015a). As such, Virgo should be an ideal environment for probing the connection between local density, dynamical history, and ICL populations in galaxy clusters.

However, a possible complication in studying the ICL in Virgo is the long line-of-sight depth of the cluster, with significant differences in the inferred depth between the various galaxy populations in Virgo. Early type galaxies appear to have a back-to-front depth of $2.4 \mathrm{Mpc}$ (Mei et al. 2007), while spirals and dwarfs show a much broader spread, with depths of $\sim 7 \mathrm{Mpc}$ (e.g., Yasuda et al. 1997, Solanes et al. 2002, Jerjen et al. 2004, although see also Cortés et al. 2008). This argues that some caution should be applied in comparing our projected ICL maps to the true three dimensional structure of the Virgo Cluster. This depth also complicates comparisons of ICL populations mapped by surface photometry to those mapped by discrete populations (planetary nebulae, red giant stars; see Mihos et al. 2009). Surface photometry integrates the ICL luminosity along the full depth of the cluster, with some minor effects due to projection (Rudick et al. 2009). In contrast, detection of discrete tracers can suffer from incompleteness bias - the detection of objects will likely be incomplete before reaching the far side of Virgo. As a result, there is a potential systematic difference between ICL estimates using the two types of techniques, in that studies using discrete ICL tracers may infer less intracluster light compared to those such as ours which employ deep surface photometry. With these caveats in mind, we now turn to a more global discussion of the intracluster light in Virgo.

Even a casual inspection of the deep imaging in Figures 1, 2, and 3 quickly reveals that much of the diffuse starlight in the Virgo Cluster is concentrated in the cluster core, around M87 and in the M86/M84 region. In areas immediately north and south of the region, there is very little diffuse light other than what can be accounted for by scattering from Galactic dust. Around subcluster B we also find little evidence for any extensive ICL component, aside from the shell system inside the halo of M49 itself. The only region in our survey area outside of Virgo's core that contains much diffuse light is the region around NGC 4365 in the $\mathrm{W}^{\prime}$ cloud, a region of high local galaxy density. The fact that the Virgo ICL is so heavily concentrated in the cluster core has also been indicated by PNe studies of fields throughout Virgo (Castro-Rodriguez et al. 2009) and is as expected from simulations of ICL production in massive clusters (Murante et al. 2004, Sommer-Larsen et al. 2005, Martel et al. 2012). While this central concentration reiterates the importance of environmental density on intracluster light production, the exact mechanism responsible for 


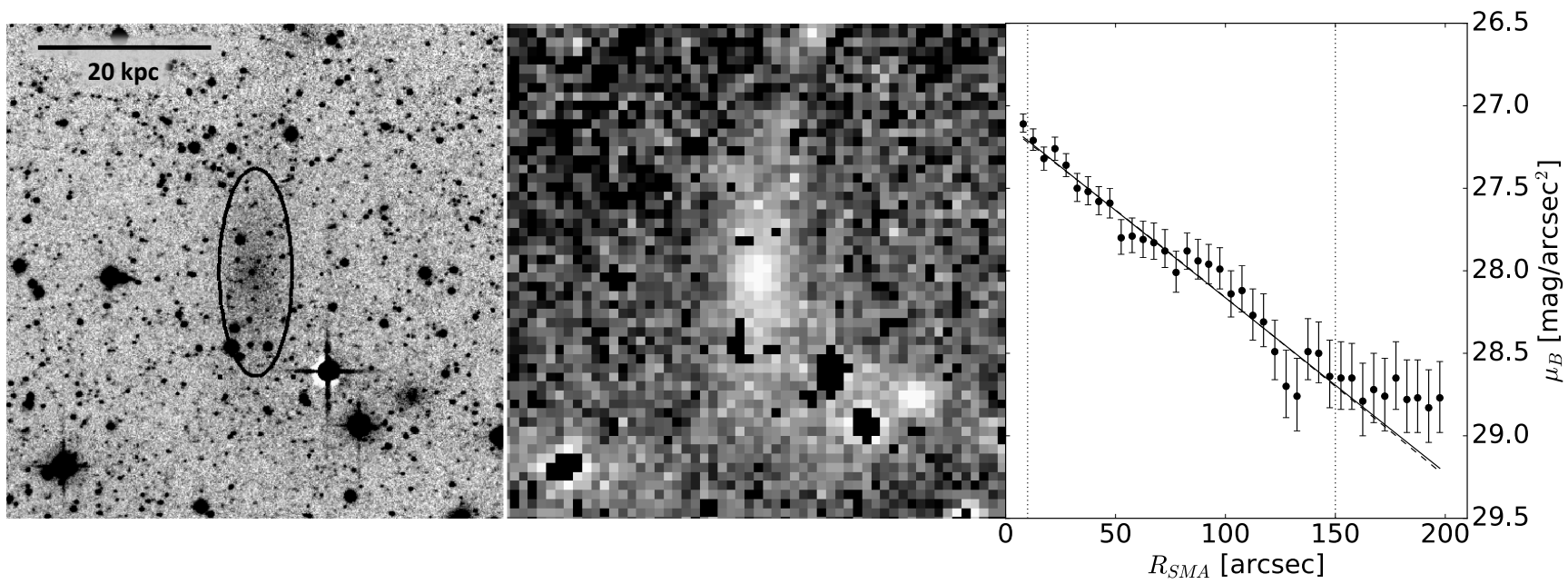

Figure 11. Left: $12^{\prime} \times 12^{\prime}$ cutout of the full resolution $B$-band Schmidt mosaic showing a new Virgo ultradiffuse galaxy. Middle: The Schmidt mosaic at the same $12^{\prime} \times 12^{\prime}$ scale, but after being masked of bright sources and median binned in $9 \times 9$ pixel blocks to show faint structure. Right: Surface brightness profile plotted as a function of semi-major axis radius, with exponential (solid) and Serśic (dashed) fits overplotted. The dotted vertical lines show radial range of the profile fits, and the outermost ellipse used in the fit is shown in the left panel.

the core ICL is not well constrained. Any of the proposed ICL production mechanisms - stripping by the central galaxy, stripping by the global cluster potential, and stripping during massive galaxy mergers - will all be most effective near the cluster center.

However, the concentration of diffuse light around NGC 4365 in the $\mathrm{W}^{\prime}$ cloud does perfectly illustrate the pre-processing role played by galaxy groups in generating the large scale diffuse intracluster light in massive galaxy clusters. The group's high local density drives strong interactions between galaxies, stripping stars out into the group environment and beginning the formation of an "intragroup halo" of light. NGC 4365 is the fifth most luminous galaxy in Virgo (Kormendy et al. 2009), only 0.3 magnitudes fainter in $V$ than M87 itself; if the $\mathrm{W}^{\prime}$ cloud was an isolated group, it might well ultimately form a massive field elliptical or "fossil group" with an extended stellar halo (D'Onghia et al. 2005, SommerLarsen et al. 2006), although the galaxy's low X-ray luminosity (Kim et al. 1992) might preclude the fossil group scenario. However, the distance and radial velocity of NGC 4365 and other members of the $\mathrm{W}^{\prime}$ cloud are consistent with the group falling into the Virgo Cluster from the cluster's far side (Mei et al. 2007); when the group eventually falls into the even denser environment of Virgo, interactions with cluster's massive galaxies and its global tidal field will strip this extended intragroup light and disperse it throughout the cluster (e.g., Mihos 2004, Rudick et al. 2006), further feeding Virgo's growing population of intracluster stars.

The large amount of diffuse light around NGC 4365 ( $\sim 3 \%$ of the total galaxy luminosity) makes the lack of ICL around M49 even more curious. Both galaxies are the central galaxies of Virgo subgroups, but they have very different diffuse light properties. In contrast to the isophotal flattening and extensive tidal structure around NGC 4365 M49's outer halo is well-behaved, showing only mild isophotal variation in ellipticity and position angle as a function of radius (Kormendy et al. 2009, Janowiecki et al. 2010), and its faint, extended shell system accounts for only $\sim 0.5 \%$ of the total galaxy light
(Janowiecki et al. 2010). The differences in the two systems may well be tied to their small-scale environment: NGC 4365 is attended by a close swarm of companion galaxies. The projected radius inside which the total companion luminosity equals half the central galaxy luminosity is $192 \mathrm{kpc}$ for NGC 4365 and $300 \mathrm{kpc}$ for M49 (235 kpc and $338 \mathrm{kpc}$, respectively, if we limit to companions with velocities within $500 \mathrm{~km} \mathrm{~s}^{-1}$ of the central's systemic velocity). The high density of companions around NGC 4365 likely results in stronger interactions and more tidal stripping from the system as compared to M49, at least at the current epoch.

Aside from the Virgo core and the $\mathrm{W}^{\prime}$ cloud, the bulk of the diffuse light we find is closely coupled to the halos of Virgo's bright ellipticals - either the substructure embedded within the halos or the extended, low density halos themselves. On these scales, separation of galaxy light from intracluster light is both difficult and ill-defined. While simulations suggest that the extended envelopes of cluster $\mathrm{cD}$ galaxies are kinematically distinct from the central regions of the galaxy (Murante et al. 2004, Dolag et al. 2010, Rudick et al. 2011) - a picture with growing observational support (Kelson et al. 2002, Gerhard et al. 2007, Ventimiglia et al. 2011, Bender et al. 2015, Longobardi et al. 2015b) - without kinematic information, any distinction becomes much less clear, as these components blend smoothly together photometrically. As much as $50 \%$ of the total cD light can be contained in this outer, diffuse envelope (Gonzalez et al. 2005) and, as illustrated by Cooper et al. (2015), much of this light comes from accretion of galaxies onto the central cD, a major mechanism associated with the formation of the intracluster light itself. Thus the extended, low density halos of the central galaxies are the accreted light that comes with the dynamical destruction of cluster galaxies, and it is no surprise that we see these extended halos around M87 and M49, the central galaxies of the Virgo A and B complexes, respectively.

Information on the processes driving ICL production in Virgo is also provided by the colors of the diffuse tidal features we see throughout the cluster. As re- 
ported here in Section 4 and also in Rudick et al. (2010), these streams are predominantly red, with $B-V$ colors in the range $0.7-0.9$. These colors suggest old stellar populations, with little evidence for young stars. These colors are also are too red to accommodate a poststarburst population — depending on the metallicity, a single burst population would take $\sim 2$ Gyr or longer to reach $B-V=0.7$ (Bruzual \& Charlot 2003), longer than the survival time of tidal tails in a cluster environment (Rudick et al. 2009). Therefore, the reddish tails we see cannot be dominated by stars formed during the interaction or in-situ in the tidal debris. While some bluer streams exist - for example, the blue tidal tails around the interacting spiral NGC 4388 (Section 4.2) or the ram pressure stripped, star-forming knots near IC 3418 (Figure 4; Hester et al. 2010, Kenney et al. 2014), the predominantly red colors of the Virgo streams argues for older, tidally stripped stellar populations as the major contributor to the intracluster light. The observed colors do not tightly constrain the stellar metallicities; for populations older than $4 \mathrm{Gyr}$, the color range $B-V=0.7-0.9$ covers a metallicity spread of of $-1.3 \lesssim[\mathrm{Fe} / \mathrm{H}] \lesssim 0.0$ (Bruzual \& Charlot 2003); a similar broad spread in metallicity was inferred from studies of intracluster red giants in Virgo (Durrell et al. 2002, Williams et al. 2007b). Given the link between mass, metallicity, and broadband colors in galaxies, the ICL colors and implied metallicity range argues that a variety of progenitor systems contribute to the ICL, from lower luminosity dwarfs to more massive systems.

The colors of the streams are somewhat bluer than the bulk of the stellar populations in the massive Virgo ellipticals, which typically have $B-V$ colors $>0.9$ in their inner regions. However, negative color gradients are common in ellipticals (as can be seen in the color maps of Figure 22, and the slightly bluer $B-V$ colors of the outer halos of M87 (Rudick et al. 2009), M49 (Mihos et al. 2013a), and NGC 4365 (this study) are similar to those of the diffuse ICL features in Virgo. Photometric modeling indicates these color gradients are most likely tracking metallicity gradients (Liu et al. 2005, Mihos et al. 2013a, Montes et al. 2014), where an old metal-rich inner halo transitions to a more metal-poor population in the galaxy outskirts and eventually into the intracluster environment (as traced by the intracluster RGB stars; Williams et al. 2007b). This similarity in color between the ICL streams and the outer halos of the massive ellipticals bolsters the argument for a common origin between these components - mergers and tidal stripping feed Virgo's ICL while also building up the halos of its elliptical galaxies.

In regard to measuring the total amount of intracluster light in our survey field, quantitative characterizations of the ICL in galaxy clusters have proved challenging to implement. An often-quoted metric is the ICL fraction, $f_{\text {ICL }}$, defined as the fraction of the total cluster luminosity contained in the ICL component. Unfortunately, such a number is both hard to define and hard to measure, due largely to the fact that there is often no clear photometric differentiation between the extended cluster ICL and the central galaxy's diffuse outer halo (e.g., Gonzalez et al. 2007, Rudick et al. 2011, Cooper et al. 2015). Simulations often define the ICL as being comprised of stars within the cluster unbound from their host galaxy (Murante et al. 2004, Dolag et al. 2010, Rudick et al. 2011, Cui et al. 2014), but lacking kinematic information for the diffuse light and detailed mass models for the cluster galaxies, this definition is rather intractable to apply. Conversely, observational definitions include measuring the total amount of light below a given surface brightness threshold (e.g., Feldmeier et al. 2004b, Burke et al. 2015), or fitting multiple components to the observed surface brightness profiles (Gonzalez et al. 2005, Krick \& Bernstein 2007, Seigar et al. 2007), but these definitions depend critically on the adopted surface brightness thresholds or the functional forms for the light profiles. Furthermore, connecting these measurements of the intracluster light distribution back to simulations of the stripped stellar mass in clusters requires additional knowledge of the stellar populations involved. Much recent progress has been made mapping out the kinematics of intracluster PNe to trace the ICL separately from the galaxy light, although mapping this back to a stellar ICL fraction requires knowledge of the underlying stellar populations in the ICL (Ventimiglia et al. 2011, Longobardi et al. 2015b, Barbosa et al. 2016). All these complications make a unique determination of the ICL fraction extraordinarily difficult (see, for example, the extensive discussions in Puchwein et al. 2010, Rudick et al. 2011, and Mihos 2015).

Nonetheless, we can at least attempt a rough estimate of the ICL fraction for Virgo using our data. The simulations of Rudick et al. (2011) show that the bulk of the cluster luminosity at surface brightnesses fainter than $\mu_{v} \sim 26-27$ (for an adopted $M / L_{*, V}=3$ ) is comprised of material unbound from its host galaxy. Furthermore, of this diffuse material, roughly $5-10 \%$ is in the form of coherent streams (Rudick et al. 2009), largely the relatively high surface brightness tidal features that represent material most recently stripped from their host galaxies. Taken together, these studies suggest that the luminosity of the observed tidal features might be used as a rough tracer of the total amount of diffuse intracluster light in Virgo, albeit with significant uncertainty given the caveats above.

In Virgo, the most luminous tidal feature we see is the SW stream around NGC 4365 in the $\mathrm{W}^{\prime}$ cloud (Figure 9), with a total luminosity of $2.3 \times 10^{9} \mathrm{~L}_{\odot, V}$. In $\mathrm{J}+10$, we also measured the luminosities of most of the discrete features around the other major ellipticals in Virgo, including M87, M86, M84, M49, and M89, which, in total, account for another $2.3 \times 10^{9} \mathrm{~L}_{\odot, V}$ of starlight. Finally, the other new streams we catalog here have a total luminosity of another $10^{9} \mathrm{~L}_{\odot, V}$, giving a total stream luminosity in our imaging survey of $L_{\text {stream }} \sim 5.6 \times 10^{9} \mathrm{~L}_{\odot, V}$. If this stream luminosity represents $5-10 \%$ of the total ICL luminosity (Rudick et al. 2009), this gives an estimate of the ICL luminosity in our field of $L_{\mathrm{ICL}}=0.56-1.12 \times 10^{11}$ $\mathrm{L}_{\odot, V}$ in our field. To turn this into an ICL fraction, we also need to know the total $V$ luminosity of all the galaxies in our survey area. To calculate this, we use the Extended Virgo Cluster Catalog (EVCC) of Kim et al. (2014), based on SDSS DR7 imaging. We transform the ugriz magnitudes to Johnson $V$ as described in the Appendix, then sum the $V$-band luminosities for all EVCC galaxies which fall in our total survey area, obtaining a total galaxy luminosity of $L_{\text {gal }}=7.1 \times 10^{11} \mathrm{~L}_{\odot, V}$. Fi- 
nally, we arrive at our final estimate for the ICL fraction of $f_{\mathrm{ICL}}=L_{\mathrm{ICL}} /\left(L_{\mathrm{gal}}+L_{\mathrm{ICL}}\right)=0.07-0.15$.

Our estimate is not without significant uncertainty, of course. We have used the simulations of Rudick et al. (2009) to estimate the rough scaling of stream luminosity to total ICL luminosity, but those simulations show significant variation in the stream-to-total luminosity ratio depending on the dynamical conditions in the cluster (see their Figure 5). If there is a relative dearth or excess of luminous ICL streams in Virgo at present, our derived total ICL luminosity will be mis-estimated. A second source of uncertainty is our irregular and limited survey footprint. Virgo's virial radius covers 104 degree $^{2}$ (Ferrarese et al. 2012), while our survey footprint only targets $\approx 16$ degree $^{2}$ in the cores of the Virgo $A$ and $B$ subclusters. How this might affect our estimate is not immediately obvious. On the one hand, simulations suggest that the ICL in clusters is more centrally concentrated than the galaxies (e.g., Murante et al. 2004; Martel et al. 2012); if so, our survey targeting the Virgo core might be biased to overestimate the total ICL fraction. On the other hand, there is likely to be significant spatial variation in amount of ICL in the cluster, as clearly demonstrated by the bright streams we observe in the $\mathrm{W}^{\prime}$ cloud. The fact that our survey does not cover several other substructures (for example, cluster $\mathrm{C}$ around M60 to the west or the M100 group to the north; see e.g., Binggeli et al. 1987), means that we may be missing ICL streams and thus underestimating the total Virgo ICL fraction. Deeper imaging over a wider field of view, such as that provided by the NGVS survey (Ferrarese et al. 2012), will be helpful in addressing this uncertainty.

Nonetheless, even with these caveats in mind, our estimated Virgo ICL fraction is in reasonable agreement with other estimates of the Virgo ICL using individual luminous stars as tracers. Ferguson, Tanvir \& von Hippel (1998) used deep HST/WFPC2 observations of intracluster red giants to derive an ICL fraction of $\sim 10 \%$ in a field $45^{\prime}$ east of M87, while a similar study by Durrell et al. (2002) in a field halfway between M87 and M86 found a somewhat higher fraction of $15 \%_{-5}^{+7}$. Using intracluster planetary nebulae, Feldmeier et al. (2004a) found a global ICL fraction of $15.8 \% \pm 3.0$ (statistical $) \pm 5.0$ (systematic) over six fields located in Virgo subclumps A \& B, while Castro-Rodriguéz et al.(2009) found a lower fraction of $\approx 7 \%$ in the cluster core, with little or no intracluster light outside of it.

Our Virgo ICL fraction is also similar to - albeit on the low end of - ICL fractions derived for other galaxy clusters. In an early compilation of estimates for nearby clusters, Ciardullo et al. (2004) showed ICL fractions spanning the range $15-35 \%$, with no clear correlation against cluster velocity dispersion or Bautz-Morgan type, a morphological classification based on the presence of a centrally dominant (cD) galaxy (Bautz \& Morgan 1970). However, some of the most spectacular examples of cluster-wide, unrelaxed intracluster light are found in clusters experiencing massive accretion events, such as Abell 1914 (Feldmeier 2004b), Hydra I (Arnaboldi et al. 2012), and Coma (Thuan \& Kormendy 1977, Gregg \& West 1998, Gerhard et al. 2007). Recent observations have pushed out to clusters at redshifts of $z \sim 0.3-0.5$ and find somewhat smaller ICL fractions,
5-20\% (Presotto et al. 2014, Montes \& Trujillo 2014, Giallongo et al. 2014), somewhat suggestive of clusters building up their ICL components over time (Burke et al. 2015, although see also Guennou et al. 2012 for a differing view). It is encouraging that our Virgo ICL fraction is consistent with measurements for other clusters; however, given the wide range measured in other clusters (5$35 \%$ ), the differing observational metrics used by these studies, and the strong variance in $f_{\mathrm{ICL}}$ as a function of metric used (Puchwein et al. 2010, Rudick et al. 2011) it would frankly be difficult not to be consistent with other measured ICL fractions.

An alternative to measuring ICL fractions in individual clusters is to photometrically "stack" imaging of many clusters; doing so washes out the cluster-to-cluster variation in streams such as those we detect in Virgo, but has the potential to push deeper in surface brightness and measure the even more diffuse underlying ICL structure. Zibetti et al. (2005) stacked SDSS data for 683 bright clusters, finding an ICL fraction of $10.9 \% \pm 5.0 \%$, with another $\approx 20 \%$ of the cluster light coming from the central BCG. Given the photometric uncertainty in decoupling the ICL from the outer BCG light, this result is in reasonable agreement with our estimate for Virgo. D'Souza et al. (2014) conducted a similar stacking study for isolated galaxies; while not directly comparable to cluster BCGs, they did find that for the most massive early type systems, comparable in luminosity to M87, 50-70\% of the galaxy light was found in a very extended, diffuse halo. If we interpret Virgo's ICL as the extended halo of its BCG and compare the total inferred ICL luminosity in Virgo to the luminosity of M87 itself $\left(1.15 \pm 0.5 \times 10^{11} \mathrm{~L}_{\odot, V}\right.$; Janowiecki et al. 2010), we find that this halo/ICL component contributes $30-50 \%$ of the total BCG+halo light. The common picture in all these comparisons is that the Virgo ICL is consistent with that measured in other clusters and around massive galaxies, although somewhat on the low end of the observed range.

The fact that the ICL fraction we infer for Virgo is on the low end of the scale compared to other clusters may be a result of Virgo's dynamical state. Simulations of galaxy clusters show that ICL fractions grow as clusters become more dynamically evolved, and can reach values as high as 40-50\% at late times (e.g., Rudick et al. 2006, 2011, Martel et al. 2012, Contini et al. 2014). However, as mentioned previously, compared to other massive clusters such as Coma, Virgo appears not as wellevolved, showing significant substructure in the spatial distribution, kinematics, and morphological types of its constituent galaxies. These various subgroups (A, B, the $\mathrm{W}^{\prime}$ cloud, and other smaller groups; see Binggeli et al. 1987) are reflective of a cluster still in the process of assembly. As the smaller groups eventually accrete into the cluster core, ICL production will increase as galaxies interact and merge in the dynamically active environment, growing Virgo's ICL component with time.

The moderately low ICL fraction we measure is likely also related to Virgo's instantaneous accretion rate we simply do not see a large number of luminous ICL streams. Luminous streams form during massive accretion events and rapidly mix away to form the more diffuse (and harder to detect) intracluster light (Rudick et al. 2006, 2009). The diffuse substructure we do observe in the extended halos of Virgo's massive ellipticals accounts 
for only $\sim 0.5-3 \%$ of the total light of the host galaxy $(\mathrm{J}+10$, this study). In the case of M87, the relatively low implied rate of accretion $\left(\sim 1 \mathrm{~L}_{\odot} \mathrm{yr}^{-1}\right.$; Section 4.1) is insufficient to build up the extended $\mathrm{cD}$ envelope in a Hubble time; instead, the accretion of more massive systems is required. Evidence for more substantial recent accretion is seen in the kinematics of globular clusters and planetary nebulae around M87 (Romanowsky et al. 2012; Longobardi et al. 2015a), and the extreme boxiness of the galaxy's outer isophotes (Section 4.1) also suggests a more violent past history. Interestingly, based on the population of globular clusters and UCDs in Virgo, Ferrarese et al. (2016) argue that in the Virgo core, as much as $40 \%$ of the present-day galaxy light may have come from disrupted satellites, again arguing for a higher rate of accretion and tidal stripping in the past. Clearly, the buildup of M87's halo (and concurrent ICL formation) is an ongoing process, but also a stochastic one - the lack of substantive photometric substructure at the present day argues that the cluster is currently in a rather quiescent phase. However, with both M84 and M86 in close proximity, and M49 and NGC 4365 both falling into the cluster, Virgo's current phase of quiet accretion is likely to end soon.

\section{SUMMARY}

We have conducted a deep imaging survey of diffuse light in the Virgo Cluster, with data taken using CWRU's Burrell Schmidt telescope over the course of seven spring observing seasons from 2004-2011. Our final survey footprint consists of 16.7 square degrees in Washington $M$ and 15.3 square degrees in a modified $B$ filter; transformed to Johnson $B$ and $V$, our $3 \sigma$ limiting surface brightnesses are $\mu_{B, \text { lim }}=29.5 \mathrm{mag} \operatorname{arcsec}^{-2}$ and $\mu_{V, \text { lim }}=28.5 \mathrm{mag} \operatorname{arcsec}^{-2}$. Having data taken in two photometric bands across multiple seasons provides not only $B-V$ colors across the field, but also gives confirmation of diffuse features detected at the faintest levels. At the depths probed by our survey we see a wide variety of diffuse structures in Virgo, including long tidal tails associated with individual cluster galaxies, the outer stellar halos of massive ellipticals, a number of large ultradiffuse galaxies, extensive intragroup light in the infalling $\mathrm{W}^{\prime}$ cloud, and several other diffuse structures having no clear association with individual galaxies.

Our survey maps also clearly reveal diffuse light arising from the scattering of Milky Way starlight by foreground galactic dust clouds, a significant source of contamination in deep imaging studies such as ours. We show that this diffuse scattered starlight is well traced by deep far-infrared $250 \mu$ Herschel imaging, and that scaling and subtracting the $250 \mu$ map from the optical map (using a scale factor of $I_{\nu}(\mathrm{V}) / I_{\nu}(250 \mu)=3.5 \times 10^{-3}$ ) yields a reasonable "dust-free" view of diffuse starlight in the Virgo Cluster.

Using our deep imaging, we identify a number of faint, extended tidal streams and plumes emanating from small galaxies in the Virgo core. Most of the streams we identify are red in color $(B-V \sim 0.7-0.9)$, suggesting that the bulk of the starlight being added to Virgo's ICL today is comprised of old stellar populations. These colors are similar to those at large radius (beyond $50 \mathrm{kpc}$ ) in M87, M49, and NGC 4365, suggesting a connection between the formation of the ICL and the growth of the outer ha- los in massive Virgo ellipticals. These observed streams are also low in luminosity $\left(\mathrm{L} \sim 10^{9} \mathrm{~L}_{\odot, V}\right)$, implying an instanteous stripping rate around $\mathrm{M} 87$ of $\sim 1 \mathrm{~L}_{\odot, V} \mathrm{yr}^{-1}$, too low to have built the galaxy's extended outer halo via accretion of small satellites over a Hubble time. Instead, a higher past accretion rate, or the accretion of more massive companions (or, likely, both) is needed, and we do find more subtle signatures of such massive accretions in the outer halos of both M87 and M86. We also show that M87's outer halo becomes extremely boxy beyond a radius of $100 \mathrm{kpc}$.

To the south of the Virgo core, in subcluster B surrounding M49, we find fewer examples of active tidal stripping, aside from the complex of accretion shells in the halo of M49 itself. We do, however, find two extended diffuse features having no clear association with nearby galaxies. One, a long $(70 \mathrm{kpc})$ diffuse stream, may trace a past encounter between M49 and NGC 4519, while the other is an amorphous patch of diffuse light $\approx 3.5^{\prime}$ in size, but of unknown origin. Aside from these features, we see no evidence for any extensive network of diffuse ICL in subcluster B. The lack of an extended ICL component in this region likely reflects its lower environmental density compared to the Virgo core around M87, as tidal stripping mechanisms operate most efficiently in regions of high galaxy density.

In contrast, in the infalling $\mathrm{W}^{\prime}$ cloud of galaxies, we find an extensive system of diffuse light around the dominant elliptical NGC 4365 and its population of satellites. The most luminous feature is the $170 \mathrm{kpc}-$ long SW Tail, which our photometry shows to have a $B-V$ color of $0.75-0.85$, similar to the outer colors of both NGC 4365 and NGC 4342, the companion galaxy projected inside the tail. We also find a thin loop of starlight to the NE of NGC 4365, along with a diffuse plume in the southeast portion of galaxy's outer halo. The large extent and total luminosity $\left(\sim 2.9 \times 10^{9} \mathrm{~L}_{\odot, V}\right)$ of the tidal debris around NGC 4365 is suggestive of strong, slow interactions between NGC 4365 and its satellites; when the $\mathrm{W}^{\prime}$ cloud is accreted into the Virgo cluster, these diffuse structures will likely be stripped from NGC 4365 and incorporated in Virgo's extended ICL component. The $\mathrm{W}^{\prime}$ cloud may thus be giving us a direct view of the pre-processing stage that galaxy groups mediate in the formation of ICL in massive clusters.

In addition to the variety of diffuse tidal structures we see within Virgo, we also find examples of large $\left(r_{e}>3\right.$ kpc), low surface brightness $\left(\langle\mu\rangle_{e, B}>28\right)$ ultradiffuse galaxies within the cluster. Three of these objects were reported in an earlier paper (VLSB-A, -B, and -C, Mihos et al. 2015), and to that list we add here a fourth: VLSB-D, a nucleated UDG located $\sim 180 \mathrm{kpc}$ north of the bright elliptical M84. The galaxy is well-fit by a Sersíc profile with index $n=0.97, r_{e}=166^{\prime \prime}(13.3 \mathrm{kpc})$, $\langle\mu\rangle_{e, B}=28.2$, and total magnitude $m_{B}=15.5$, making it one of the most extreme UDGs yet detected in any galaxy cluster. The galaxy is quite elongated and shows evidence of extended tidal debris, and may be in the process of being disrupted by the cluster environment. Like its cousin VLSB-A (Mihos et al. 2015), in VLSB-D we may be witnessing undergoing a tranformation from a nucleated LSB galaxy to a ultracompact dwarf, once the galaxy has been completely disrupted and only its 
compact nucleus remains intact.

Taken as a whole, our observations show a cluster still in the process of growing its intracluster light component. Using the tidal streamers as a tracer of the even more diffuse ICL below our detection limit, we estimate an intracluster light fraction in the range $7-15 \%$, similar to other estimates of the Virgo ICL fraction (Ferguson et al. 1998, Durrell et al. 2002, Feldmeier et al. 2004a, Castro-Rodriguéz et al. (2009). However, this value falls in the lower end of measured ICL fractions in other massive clusters, and is also smaller than expected for fully evolved clusters, which simulations suggest should possess ICL fractions as high as 40-50\% (e.g., Rudick et al. 2009, 2011, Martel et al. 2012, Contini et al. 2014). Virgo's relatively low ICL fraction is likely a function of its dynamical state - the cluster shows significant substructure and is still in the process of assembling. As Virgo's various subgroups are accreted into the main cluster core, the amount of intracluster light will continue to grow, as the diffuse shells and streams around M49 and NGC 4365 are incorporated into M87's outer halo and Virgo's extended ICL component, while additional starlight will be stripped from galaxies during the strong interactions that that accompany subcluster mergers.

With the survey complete, we are making the imaging data publicly available. The full resolution $B$ and
$M$ mosaics, along with the masked/rebinned low surface brightness maps, are available as FITS files for download at http://astroweb.cwru.edu/VirgoSurvey/.

We thank Pat Durrell for useful discussions, along with Laura Ferrarese, Pat Côté, Eric Peng, and other members of the Next Generation Virgo Cluster Survey team. We also thank Charley Knox for his tireless assistance with the mechanical, optical, and IT systems at the observatory. Over the course of this project, J.C.M. has been supported by the NSF through grants AST-9876143, ASTR-0607526, AST-0707793, and AST1108964, as well as by the Research Corporation through a Cottrell Scholarship. J.J.F. has been supported by the NSF through grants AST-0302030 and AST-0807873, as well as by the Research Corporation through a Cottrell College Science Award (7732).

This research has made use of NASA's Astrophysics Data System Bibliographic Services. The authors further acknowledge use of the NASA/IPAC Extragalactic Database (NED), which is operated by the Jet Propulsion Laboratory, California Institute of Technology, under contract with the National Aeronautics and Space Administration.

Facility: CWRU:Schmidt

\section{APPENDIX}

We begin this section with a general description of the Burrell Schmidt and the modifications made to enhance its capabilities for deep surface photometry. We then follow with an overview of our general observing strategy and a description of each season's observing, noting changes to the telescope system during the course of the survey. Finally, we detail our process of data reduction and construction of the final photometrically-calibrated images.

\section{A.1 THE BURRELL SCHMIDT}

The Burrell Schmidt is a Case Western Reserve University owned and operated wide-field Schmidt telescope currently situated at Kitt Peak National Observatory. Its 24-inch corrector and 36-inch primary mirror deliver a f/3.5 beam resulting in a plate scale of $97.2^{\prime \prime} \mathrm{mm}^{-1}$ (Nassau 1945). Two different CCDs were in use on the telescope over the course of the Virgo survey (see below); each employed 15 micron pixels, yielding a pixel scale of $1.45^{\prime \prime}$ pixel ${ }^{-1}$. Depending on the CCD in use, the field of view of the camera was either $0.825^{\circ} \times 1.65^{\circ}(2004-2007)$ or $1.65^{\circ} \times 1.65^{\circ}$ (2008-2011). While the large pixel scale undersamples the stellar point spread function of the Schmidt, for our survey we are more interested in diffuse surface photometry rather than point source photometry, and the $\mathrm{f} / 3.5 \mathrm{beam}$ works to our advantage here, delivering a wide field of view onto a single CCD.

A second advantage for the Burrell is that it has a closed, well-baffled telescope tube. This design minimizes scattered light from off-axis sources, a potentially serious source of contamination in deep surface photometry (see, e.g., Feldmeier et al. 2002). We also cover the interior of the telescope tube and the telescope baffling using black Protostar flocking with very low reflectivity $(<0.7 \%)$ to minimize off-axis light from scattering off the interior of the telescope and reaching the CCD (see, e.g., Pompea et al. 1989).

Reflections between elements in the optical path can also be a source of image contamination, and here again the Schmidt layout works to our advantage. The small aperture and fast beam work to deliver a wide field of view without the need for complicated reimaging or corrective optics, leaving an optical path with very few elements: corrector, primary, Newtonian flat, filter, and dewar window. In most seasons (see section A.3), the filter and dewar window were coated with anti-reflection (AR) coatings with reflectivities $R<0.5 \%$ (and more typically $R \sim 0.2 \%$ ), matched to the central wavelength of the filter in use. These reflectivities are significantly less than typical broad-band $\mathrm{MgFl}$ AR coatings $(R \sim 1.5 \%)$, and significantly reduce contamination in the images. Furthermore, we can measure and remove the low level stellar reflections that remain (see $\S$ A.4 below). As the CCD itself is the most reflective element in the system (other than the primary and Newtonian mirrors), the brightest reflections involve bounces between the CCD and the surfaces of the dewar window and filter, producing halos of radius $5^{\prime}$ and $19^{\prime}$, respectively. In total, these halos typically contain $<0.5 \%$ of the light from the star, and we have developed a technique for their efficient removal from the images (see Slater et al. 2009). The only residual feature left behind is a bright pair of rings at $r \sim$ $1^{\prime}$ and $2^{\prime}$ due to reflections within the corrector itself as light first enters the telescope. More complicated reflections involving multiple bounces between surfaces blend into the extended profile without leaving distinct features, and so are removed via subtraction of the PSF. 
Schmidt telescopes also possess a unique reflection (the "Schmidt ghost") formed by light which reflects off the CCD, goes back up the telescope tube, hits the corrector lens, and reflects back down to the CCD on the opposite side of the optical axis (see, for example, Yang, Zhu, \& Song 2002). This results in complicated ghosts from bright stars, displaced opposite the optical axis from the parent star. For bright stars, we mask the brightest part of the ghost (an irregular feature approximately $1.5^{\prime}$ in size) in the data reduction phase. For the more extended, low surface brightness portion of the ghost, we take advantage of the fact that its position with respect to its star changes as the star's position on the chip changes. Therefore, a combination of images taken with large dithers minimizes any small feature that it might otherwise imprint on our data.

To increase the stability of the flat fielding, over the course of the survey we made several modifications to the support structure for the Newtonian secondary mirror. This is important since any flexure in the support can lead to illumination differences in the image plane, particularly if the secondary mirror is undersized and the full beam is not reflected over the full field of view. Prior to the start of the survey, we re-engineered the telescope to move the Newtonian further up the telescope tube so that the Newtonian was not overfilled by the beam, while also moving the focal plane inwards to compensate. In 2007, we redesigned the Newtonian support from a one-armed mount to a traditional four-sided spider design. A larger Newtonian mirror was also installed, supported by three Invar flexures glued to the rear of the mirror. This mount prevents light scattering from the mirror cell onto the CCD. Baffling is installed above the Newtonian mirror as well to prevent incoming light from the corrector illuminating the rear of the Newtonian mirror. All these modifications significantly improved the stability of the support and reduced scattered light inside the tube.

As noted previously, the CCD imager went through several changes during the lifetime of the survey. The first generation CCD, in use from 2004-2006, was a SITe ST-002A 2048×4096 back-illuminated CCD operated with a gain of $2.3 e^{-} / \mathrm{ADU}$ and readnoise of $6.5 e^{-}$. In 2007, we installed two of these CCDs in the image plane, but unfortunately one failed, leaving us again with a single $2048 \times 4096$ CCD. There were also grounding problems with this system, which led to increased electronic noise - the effective readnoise for the CCD climbed to $\sim 10-20 e^{-}$. This system was replaced in 2008 with a new STA0500A $4 \mathrm{~K} \times 4 \mathrm{~K}$ back-illuminated CCD from the University of Arizona's Imaging Technology Lab, operated with a gain of $2.7 e^{-} /$ADU and readnoise of $4.0 e^{-}$. In all seasons we use a Leach controller (Leach \& Low 2000) and the Voodoo data acquisition software, under which each $2 \mathrm{~K} \times 4 \mathrm{~K}$ chip was read out through two amplifiers, while the $4 \mathrm{~K} \times 4 \mathrm{~K}$ chip was read out through four amplifiers. The CCD bias frames typically show high frequency structure (at the $\sim 1-2$ ADU level) within 25 pixels of the CCD edges which appear to change slightly with temperature. We mask these regions of the chip during data process to avoid this additional noise source.

\section{A.2. OBSERVING STRATEGY}

Over the course of the survey, spring observations with the Burrell Schmidt concentrated on the Virgo Cluster. Between two and four dark runs each season were dedicated to this project, with each run lasting 8-12 nights. At the beginning the first run of each season, the CCD dewar was equipped with a dewar window whose AR coating was specifically matched to wavelength of the filter used in that season's observations. The CCD was then mounted and aligned on the telescope, and the filter install in the filter wheel. Once this process was complete, no further changes to the system were permitted for the rest of the observing season. This includes filter changes - the filter wheel position was held fixed for the season, and all observations are made in one filter. This limits variations in the flat fielding due to slight changes in either the optical alignment of the CCD or the position of the filter, and reduces dust on the optical surfaces that can adversely affect the flat fielding.

We chose the filters used for the survey by balancing the need to maximize signal from the intracluster stellar populations while minimizing noise in the background sky. ICL populations are expected to be predominantly old, such that their starlight should peak in the red. However, the night sky in the red is both brighter and more highly variable than it is in the blue, due to strong emission lines from oxygen and $\mathrm{OH}$ molecules in the atmosphere (see, e.g., Krisciunas et al. 2007, Patat 2008, Neugent \& Massey 2010). We therefore conducted survey observations from 20042008 using the Washington $M$ filter, a broadband filter similar in width to Johnson $V$, but $\sim 250 \AA$ bluer. Washington $M$ avoids the bright and variable O I $\lambda 5577$ night sky emission line present in the redder Johnson $V$ filter (see Figure 1 of Feldmeier 2002), and is thus in the "sweet spot" to balance the competing demands of red starlight and a quiet blue sky. We then switched to a modified $B$ filter for the 2009 and 2011 seasons, re-imaging the Virgo core and M49 fields to obtain $B-V$ colors for the diffuse ICL in Virgo. Because the Washington $M$ filter is bluewards of Johnson $V$, to ensure we had a sufficient spectral baseline to obtain meaningful colors, the $B$ filter we use is bluer than standard Johnson $B$ by $\sim 200 \AA$. During data reduction, all magnitudes are transformed into standard Johnson $B$ and $V$ (see the photometric calibration discussion below), and for simplicity we refer to the data as our $B$ and $V$ imaging with the understanding that the filters we use are not actually standard Johnson $B$ and $V$ filters.

To achieve the $\sim 0.1-0.3 \%$ accuracy we aim for in the large scale flat fielding to allow measurements of diffuse light at low surface brightness, dome or twilight flats are insufficient. Dome flats illuminate the CCD with light much different in color than the night sky, introducing color-dependant systematic uncertainties into the flat, while twilight skies are much bluer and do not uniformly illuminate the large field of view of the CCD due to the illumination gradient in the twilight sky. Instead, we use night sky flats, built from a large number of blank sky pointings taken adjacent in time and at similar telescope position to the Virgo pointings (see, e.g., Morrison et al. 1997; Feldmeier et al. 2002; Rudick et al. 2010). A typical observing pattern was two sky frames an hour west of Virgo, four pointings in Virgo, and two sky pointings an hour east of Virgo which, given our exposure times of 900 s or 1200 s in $M$ and $B$, respectively, ensures 
that the skies are taken at the same hour angle (and declination) as the Virgo observations. Flats built this way will thus consist of skies taken under similar conditions to the Virgo pointing, minimizing systematics in the flat field due to different gravity loading of the camera/telescope. The color of the Kitt Peak night sky $(B-V \approx 0.9 ;$ Neugent \& Massey 2010) is also similar to the expected color of the ICL, further reducing systematic uncertainties in surface photometry of the ICL.

We took data only during photometric nights with the moon below the horizon. By observing with the moon down, we significantly reduce both the overall sky brightness as well as the presence of sky gradients due to scattered moonlight. The requirement for photometric conditions guards against artificial light from the ground (most notably from Tucson to the northeast) scattering off cirrus clouds and masquerading as diffuse light. Furthermore, even thin cirrus will scatter starlight and introduce extended halos around bright stars which can swamp the diffuse ICL we are trying to measure. Therefore, our photometric requirement was quite stringent; the scatter in frame-by-frame photometric zeropoints in our data was typically 1\%, except for 2007 where weather conditions forced us to relax our criterion somewhat (see Table 2. We also limited all observations to be taken at zenith distances less than $50^{\circ}$ (airmasses less than 1.5), which reduces both the absolute sky levels and any gradients in the sky even further. We do however need sufficient counts in the blank sky images to build accurate night sky flats. During dark time, the Kitt Peak sky surface brightness at zenith is roughly 22.8 and $21.9 \mathrm{mag} \operatorname{arcsec}^{-2}$ in $B$ and $V$ respectively (Neugent \& Massey 2010) but shows variation of half a magnitude or more, with dependencies on time of night, telescope pointing, and solar cycle (Neugent \& Massey 2010, see also Krisciunus et al. 2007, Patat 2008). Our observations span the solar minimum between cycles 24 and 25, and the observed sky brightnesses ranged from $\mu_{B}=22.4-21.8$ and $\mu_{V}=21.9-21.2$. The exposure times for the blank sky pointings (1200s and 900s in $B$ and $M$, identical to the Virgo exposures) thus resulted in instrumental sky levels of 700-1000 ADU and 900-1500 ADU in $B$ and $M$ respectively, giving us a per-pixel signal-to-noise of $\sim 2 \%$. Combining $50+$ such blank sky fields together thus delivered the $<0.3 \%$ accuracy we seek.

In each season we concentrated our imaging on a different subfield of the Virgo Cluster (see Table 1), chosen both for scientific merit and to avoid, as much as possible, contamination from Galactic cirrus. In each field, we took 30-100 images, dithering the individual images by up to half a degree. By dithering on such large scales, we reduced the effect of large scale flat fielding uncertainties, since in the final mosaic any particular object will have been imaged multiple times on a variety of positions on the CCD. The large dithering also combats contamination from the Schmidt ghost, and widens the areal coverage of our survey. Because of this seasonal targeting and dithering strategy, the full area of the survey has an effective exposure time which varies across the field; the final exposure maps in $M$ and $B$ are shown in Figure 12 .

\section{A.3. SEASON SPECIFIC OBSERVING NOTES}

Spring 2004 - In the first season of imaging, we imaged the core of the Virgo cluster in the $M$ filter in two fields covering approximately $2.25 \mathrm{deg}^{2}$. These fields included the bright ellipticals M87, M86, and M84, and a reduction of this field was presented in Mihos et al. (2005). In 17 nights over three observing runs, we took 72 images of the Virgo core, with 63 flanking sky fields for building the night sky flat. We also took 70 images of the Hercules cluster for a different project, along with 64 flanking skies. Because Hercules and Virgo are at very similar declination, the Hercules skies are similar in pointing to the Virgo skies, and we combined both sets of skies (127 images) to build the final flat. In this season, both the $M$ filter and the flat dewar window had standard MgFl antireflection coatings, giving rather strong reflections around the stars that were later removed in the data reduction stage.

Spring 2005 - In Spring 2005 we imaged three fields in the $M$ filter - one to the north of the Virgo core, one to the southeast of (and including) M87 and one northeast of (and including) M49. Over two observing runs we obtained 134 images of Virgo and 103 flanking fields in 15 nights of observing. Prior to the start of the season, both the primary and Newtonian mirrors were realuminized, improving throughput and reducing scattered light.

Spring 2006 - In this season, we imaged a field southwest of M49 in the $M$ filter. We had 16 nights of data in two observing runs, during which we took 93 images of Virgo and 78 flanking skies. This season also saw the installation of a combined dewar window / field flattener to improve the image quality across the large field of view of the CCD. Both the dewar window and the $M$ filter were coated with low reflectivity AR coatings, reducing stellar reflections.

Spring 2007 - In 2007, we upgraded the Newtonian mount and mirror and also replaced the single $2048 \times 4096$ CCD with two identical $2048 \times 4096$ CCDs butted together. However, as noted in $\S$ A.2, electronics problems compromised the CCD system - only one of the two CCDs were operational, and it showed increased electronic noise. Nonetheless, even though the data quality suffered, we still imaged fields southwest of M87 and northwest of M49 in the $M$ filter. The data was taken in 16 nights spread over three observing runs, and consisted of 111 images of Virgo and 92 flanking skies.

Spring 2008 - In Spring 2008, the $4096 \times 4096$ CCD was installed, giving us the wider field of view. However, when cooled, this chip curved by more than half the radius of curvature of (and in the same direction as) the Schmidt focal plane. Better image quality was achieved by re-installing the flat dewar window (with standard MgFl coating). The Newtonian mirror was also realuminized at this time. This engineering work gave us less observing time during the season, but in one science observing run using the $M$ filter, we obtained 8 nights of data and acquired 51 images of a field southwest of M87, along with 61 flanking skies. 

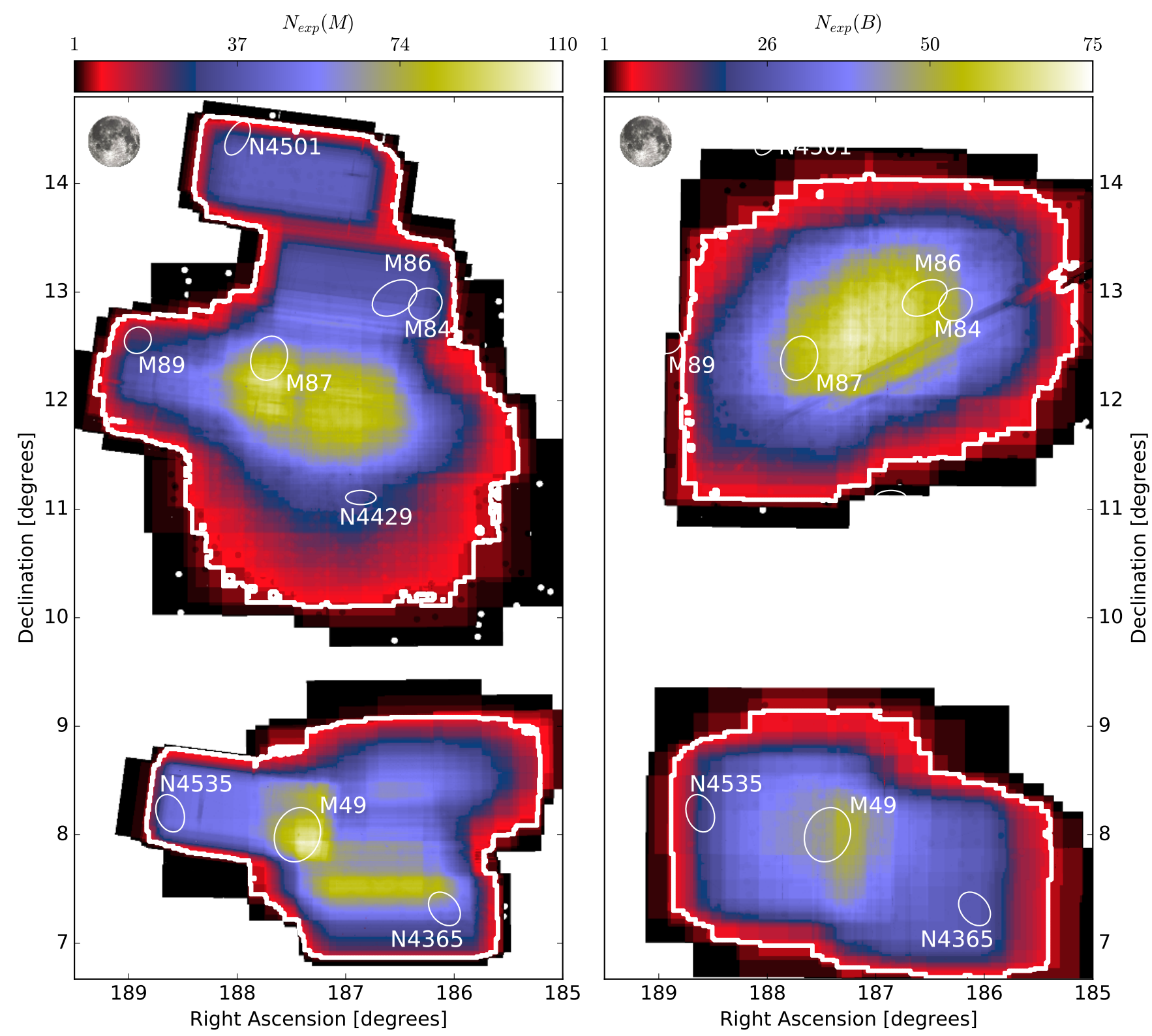

Figure 12. Exposure maps showing the number of images contributing to the final Virgo mosaics in Washington $M$ (left) and modified Johnson $B$ (right). Orientation and scale are as in Figures 13 The white contours show the minimum exposure boundary of 5 images (100 and 75 minutes in $B$ and $M$, respectively) that defines our official survey area. In the $B$-band exposure map, the diagonal striping is due to the masking of a small comet that moved through the field during the observing season.

Spring 2009 - In Spring 2009, we re-imaged the Virgo core field of Spring 2004 in the $B$ filter. In three runs we had 24 usable nights, during which we collected 102 Virgo images and 116 flanking skies. With the larger $4 \mathrm{~K} \times 4 \mathrm{~K}$ CCD, we were able to cover a field much larger in area than the original Spring 2004 core field. Before this season, we also realuminized the primary mirror. The $B$ filter was coated with a very low reflectivity AR coating which almost completely eliminated reflections off the filter; however we again used the flat MgFl-coated dewar window. Finally, during this run a comet $(65 \mathrm{P} / \mathrm{Gunn})$ passed through the Virgo cluster, forcing us to aggressively mask the comet and its tail during the data reduction phase so that it would not contaminate our measurements of the diffuse light in Virgo.

Spring 2011 - In Spring 2011, we reimaged the region around M49 in the $B$ filter. Over the course of three runs, we obtained 71 Virgo images and 73 flanking skies. In this season we also reinstalled a new flat dewar window with the aggresive AR coatings matched in wavelength to our $B$ filter. 
Table 1

Observational Datasets

\begin{tabular}{|c|c|c|c|c|c|}
\hline Season & Filter & CCD & Fields & $\mathrm{N}_{\text {Virgo }}$ & $\mathrm{N}_{\text {sky }}$ \\
\hline 2004 & $\mathrm{M}$ & $2 \mathrm{~K} \times 4 \mathrm{~K}$ & Core & 72 & 127 \\
\hline 2005 & $\mathrm{M}$ & $2 \mathrm{~K} \times 4 \mathrm{~K}$ & North, M87SE, M49NE & 134 & 103 \\
\hline 2006 & $\mathrm{M}$ & $2 \mathrm{~K} \times 4 \mathrm{~K}$ & M49SW & 93 & 78 \\
\hline 2007 & $\mathrm{M}$ & $2 \mathrm{~K} \times 4 \mathrm{~K}$ & M87SW, M49NW & 111 & 92 \\
\hline 2008 & $\mathrm{M}$ & $4 \mathrm{~K} \times 4 \mathrm{~K}$ & M87SW & 51 & 61 \\
\hline 2009 & $\mathrm{~B}$ & $4 \mathrm{~K} \times 4 \mathrm{~K}$ & Core & 102 & 116 \\
\hline 2011 & $\mathrm{~B}$ & $4 \mathrm{~K} \times 4 \mathrm{~K}$ & M49 & 71 & 73 \\
\hline
\end{tabular}

\section{A.4. DATA REDUCTION}

\section{A.4.1 Initial Steps}

We reduced the data for each observing season separately, and only combine datasets during the construction of the final mosaic. We began data reduction for each image by subtracting overscan in both directions along the CCD, followed by subtraction of a nightly master zero image created from a median of 25 individual zero frames taken at the beginning of each night. These master zeros typically showed very little structure at the $\sim 1$ ADU level (a level which corresponds to surface brightnesses of $\mu_{B}=30.4$ and $\mu_{V}=29.6$ in individual images). For observations in the 2008, 2009, and 2011 seasons, this step was followed by a correction for crosstalk in the CCD amplifiers (at the $\approx$ $0.04 \%$ level), an artifact that was not present in the earlier CCD electronics.

\section{A.4.2 Flat Fielding}

The next step of the process was to construct the night sky flat field from a combination of the individual flanking sky images (see Morrison et al. 1997, Feldmeier et al. 2002, and Rudick et al. 2010 for full details of this process). Sky pointings are chosen to have as few bright stars as possible; nonetheless with such a wide field of view, the presence of some bright stars and background galaxies is inevitable. These objects, along with large scale gradients in the night sky brightness, can introduce noise into the flat field. To reduce the influence of these features, we begin by masking bright or extended objects in the sky fields. For each sky image, we use IRAF's objmasks task to construct a mask of all features $3.5 \sigma$ above the local sky; this mask is then used as the input mask for a second run of objmasks, which results in a better sky estimate and allows for fainter objects to be masked. After this double run of objmasks, the sky images are binned up $32 \times 32$ and and examined by eye to search for very low surface brightness extended features such as the wings of bright stars or the presence of galactic cirrus. These features are then masked by hand or, in cases where the scattered light from the infrared cirrus contaminated too much of the image, the image is removed from the process entirely.

Once masked, the sky images still contain mild illumination gradients caused by night sky variations, which we remove in an iterative fashion. First, the skies are median-combined to make a preliminary master flat field. This preliminary flat is applied to each of the sky images, which are then masked and binned $32 \times 32$ and fit for any residual sky plane. These planes are subtracted from the skies, and the plane-subtracted skies are then median-combined to make a new flat field, and the process of plane fitting is repeated. We repeat this process five times, after which the residual plane fits are typically consistent with zero.

We test the uncertainty in the seasonal flats by comparing flats constructed of independent subsets of sky images. The largest noticeable difference came from comparing flats constructed from data taken during different observing runs. A division of flats from different runs often showed an edge-to-edge gradient of $\lesssim 0.5 \%$, which, if left uncorrected, could imprint residual sky variation at the $\mu_{V} \sim 27.6, \mu_{B} \sim 28.0$ level. However, these gradients were typically well modeled by a plane, and our usual strategy was to create a master flat from all the data in a given season, and then fit and apply a planar correction term for each observing run to correct them to the seasonal flat. In two seasons 2007 and 2009 - the run-to-run flat comparison showed more spatial variations across the chip, an effect likely due to known problems controlling the CCD temperature during these seasons. For these seasons, we construct run-specific flat fields to reduce the data.

\section{A.4.3 Photometric calibration}

After flat-fielding the Virgo images, we calculate the photometric zeropoint directly for each frame using SDSS photometry for the 100-200 SDSS stars brighter than $\mathrm{g}=16$ typically found in each field. SDSS ugriz photometry is transformed to Johnson $B$ and $V$ using the prescription of Lupton, who matched SDSS Data Release 4 photometry with Stetson's photometric standard\$? Lupton's transformation is given by:

$$
\begin{aligned}
& B=g+0.3130(g-r)+0.2271, \\
& V=g-0.5784(g-r)-0.0038
\end{aligned}
$$

9 The Stetson standards are closely tied to the Landolt standard system, and available at http://www.cadc-ccda.hia-iha.nrc- 
Table 2

Photometric Calibration

\begin{tabular}{lcccc}
\hline \hline Season & Filter & $C$ & $K$ & $\sigma(Z P)^{\mathrm{a}}$ \\
\hline 2004 & M & 0.212 & 0.131 & 0.012 \\
2005 & M & 0.216 & 0.148 & 0.167 \\
2006 & M & 0.219 & 0.153 & 0.008 \\
2007 & M & 0.284 & 0.174 & 0.028 \\
2008 & M & 0.297 & 0.316 & 0.006 \\
2009 & B & 0.108 & 0.274 & 0.006 \\
2011 & B & 0.142 & & \\
\hline a
\end{tabular}

a $\sigma(Z P)$ is the dispersion in photometric zeropoints for each season's images after correction for airmass and nightly zeropoint offsets.

Lupton found that the residuals from these relationships were small $\left(\sigma_{B}=0.011, \sigma_{V}=0.005\right)$, although it should be remembered that the SDSS DR4 footprint was restricted to high-latitude regions, so there are very few stars with $B-V$ less than 0.4 in the data he used. Comparisons with other $B-V$ data on the Landolt system for populations containing bluer stars (Morrison et al., in preparation) show that Luptons transformations may produce $B-V$ values which are systematically up to 0.05 magnitudes too red for stars bluer than $B-V=0.4$. There are also some small effects due to luminosity and metallicity which occur for redder stars $(B-V>1.0)$, but they should be of order 0.02 magnitudes or smaller for stars in the color range typical of intracluster light.

We then fit our instrumental aperture photometry to the SDSS-derived $B$ and $V$ magnitudes via the expressions:

$$
m_{i}=m_{\text {inst }}+C_{i}(B-V)+K_{i} X+Z P_{\text {frame }}
$$

where $i$ refers to the season-specific filter, $m_{i}$ and $B-V$ are the SDSS-derived magnitude and color of the star, $m_{\text {inst }}$ is our instrumental magnitude, $C_{i}$ is the color term, $K_{i}$ is the extinction term, $X$ is the airmass, and $Z P_{\text {frame }}$ is the frame zeropoint. Table 2 gives the parameters for the photometric solutions for each season, while Figure 13 shows the frame-to-frame variation in photometric zeropoint.

\section{A.4.4 Star Subtraction and PSF Effects}

Once the photometric solution has been established, the images are ready for star subtraction, a process which involves removing both the reflection halos and extended PSF wings of stars on the images. This star subtraction must be applied on an image-by-image basis, rather than on the final mosaic, for the following reasons. First, the position of the reflections with respect to their parent star changes as a function of the star's position on the CCD, and the dithering of the pointings means that the reflections will differ from image to image. Second, accurate sky levels must be modeled and removed prior to constructing the final mosaics, and if not removed, the extended wings of stars can lead to systematic errors in the determination of these sky levels.

Our technique for star subtraction is detailed at length in Slater et al. (2009), and we give only an overview here. Using a series of deep (450-1200s) images of the bright stars Regulus and Arcturus, we make a model for the intensity and position of reflections between the CCD, dewar window, and filter as a function of the star's position on the CCD. After subtracting out these reflections, we characterize the extended stellar PSF out to $1^{\circ}$ scales using a 5th order polynomial. We then also take a series of short $(90 \mathrm{~s}, 10 \mathrm{~s}$, and $1 \mathrm{~s})$ exposures of the Virgo fields to obtain accurate instrumental magnitudes for the bright $\left(m_{V}=8-12\right)$ stars in our target fields. Armed with accurate magnitudes and a well-determined model for stellar reflections and the PSF, we can subtract the extended halos of these stars from each of the Virgo images as detailed in Slater et al. (2009).

One step we do not take is to attempt any deconvolution of the images using the measured Schmidt PSF. In some telescope systems, the instrumental PSF can scatter a significant amount of light outwards from the inner regions of a galaxy, artificially changing the surface brightness profile and colors of the low surface brightness outskirts (see Sandin 2014, 2015, Duc et al. 2015, Trujillo \& Fliri 2016 for recent discussions). A variety of arguments suggest this effect is minimal across our survey area down to our limiting magnitudes. First, the aggressive AR coatings used on our filters and dewar window limit the bright halos of scattered light that plague data taken on systems using standard $\mathrm{MgFl}$ coatings. Second, scattered light from the PSF would tend to make the outer isophotes of bright ellipticals rounder at large radius, yet we observe the opposite - higher ellipticities at large radius (Janowiecki et al. 2010, this paper). Third, our updated reconstructions of the Burrell Schmidt PSF (Watkins et al. 2016) extend to $1^{\circ}$ and show that the reflections and extended wings of the PSF are slightly brighter in $M$ than in $B$. This argues that the scattered galaxy light would be red, in contrast to the color profiles we derive for bright Virgo ellipticals which show modest blueward gradients (Rudick et al. 2010, Mihos et al. 2013a, this paper). We also find no evidence that the Burrell Schmidt produces consistently biased colors at low surface brightness - in other studies, we have found a variety of color gradients in galaxy outskirts, both red and blue (Mihos et al. 2013b, Watkins et al. 2014, 2016). Fourth, detailed modeling of the effect using the Burrell Schmidt PSF shows that color biases of 0.1 magnitudes or more show up only below surface brightnesses of $\mu_{B} \sim 28.0$, where the color uncertainty is dominated by other sources of error such as background sources and sky subtraction (Watkins et al. 2016). Finally, since any such biases only occur in the outskirts of bright galaxies, the bulk of the intracluster environment we survey should be uninfluenced by scattered light from bright galaxies in the field. 


\section{A.4.5 Sky Subtraction}

Accurate sky subtraction is critical for quantitative surface photometry at faint surface brightnesses. The problem of sky subtraction is particularly difficult in our imaging, for a number of reasons. First, the angular scale of the Virgo cluster is so large that our images never extend outside the cluster; obtaining a "non-Virgo background" is impossible. Second, many of the Virgo galaxies themselves so large that their extended low surface bright envelopes dominate the field of view of a single image. Lastly, regions of Virgo are contaminated by galactic cirrus, which produces an extended component of diffuse scattered galactic light in the images. These features begin to show up at surface brightnesses of $\mu_{V} \sim 27$, corresponding to $\sim 8$ ADU in our imaging - comparable to the gradients in the night sky across a single image. With all these sources of diffuse emission in our Virgo fields, it is often difficult to find regions of "pure sky" in our images with which we can determine the absolute sky level and measure sky gradients. As a result, sky subtraction is particularly problematic in our imaging, and is the largest source of uncertainty in doing absolute photometry over large angular scales in our final mosaics.

To alleviate these issues as much as possible, we first use a double application of objmasks to aggressively mask sources - Virgo galaxies, background objects, satellite trails, and star subtraction residuals - in each star subtracted image. Even this masking leaves behind the extended low surface brightness envelopes of the giant ellipticals (most notably M87, M86, M84, and M49). We next use the isophotal fits of Janowiecki et al. 2010 - based on an earlier reduction of our imaging - to define elliptical masks around these galaxies out to a radius where their light profiles drop below 1 ADU. After these masks are applied, we median bin the images into 32x32 pixel bins, and use these binned images to fit the night sky background across the image. Because of the presence of very low surface brightness residual structure in our images - due to galactic cirrus, extended galaxy halos, and possible gradients in the underlying ICL itself - high order non-linear fits could be unduly biased by the spatial distribution of these features, and subtracting such sky fits run the risk of imprinting artificial structure onto our reduced images, or possibly removing true Virgo ICL. Therefore, we take a conservative approach and only fit planes to the sky background for removal.

Finally, we note that in Mihos et al. (2005) we used an iterative method for fitting skies which mapped the deviations of each sky-subtracted image from the median of the the ensemble to determine subsequent corrections to the fitted sky plane. Subsequent testing has shown this technique does not result in significantly improved sky fits, and under certain conditions it can drive the plane fits away from convergence. In the final data reduction pipeline detailed here, we take the more conservative and simple approach of only fitting a single plane to each image to subtract as sky.

\section{A.4.6 Final Mosaicing}

To build the final mosaic, we first create a series of six $6750 \times 6750$ pixel master subimages that spatially cover the Virgo pointings and have a pixel scale matched to that of the original images $\left(1.45^{\prime \prime}\right.$ pixel $\left.^{-1}\right)$. For each of these subimages, we use IRAF's wregister task to register the individual images to the master subimage, then scale them to a common photometric zeropoint. We then median combine all the individual images together into the master mosaics using imcombine with a $3 \sigma$ rejection. For this final combine, we mask any obvious artifacts on the images, such as glints from bright stars which fell on the edge of the CCD, and we also conservatively mask pixels within 100 pixels of the CCD edges to avoid the temperature-sensitive "edge noise" mentioned in Section A.1.

In order to probe the faintest surface brightnesses possible, once the final mosaics have been constructed, we spatially rebin the mosaics over larger area, medianing the pixel values over these larger scales. Before rebinning, however, we mask the images of discrete sources such as stars, bright galaxies, and fainter background objects which would otherwise bias the measurement of diffuse light on larger scales. The masking takes place in several steps. First, we use IRAF's rmedian task to run a ring median of size 10 pixels $\left(14.5^{\prime \prime}\right)$ across each mosaic, then subtract this ring median image from the original to produce an image akin to an unsharp masked image which emphasizes discrete sources on small scales. We then run the objmask task on this image, detecting objects which are $5 \sigma$ above the background level. Pixels in objects detected this way are then masked in the original mosaics. We then spatially rebin the mosaics in $9 \times 9$ pixel $\left(13^{\prime \prime} \times 13^{\prime \prime}\right)$ bins, calculating the median intensity of unmasked pixels in each spatial bin to create. During this process, spatial bins in which more than $50 \%$ of the pixels are masked are themselves masked. The deep masked/binned images shown in Figure 1, 2, and 3b have all been processed this way.

Finally, to compute the final photometric solution for the mosaics, we made a number of simplifying assumptions. Because the color terms for the photometric solution varied in each season, we simply adopt an average color term of $C_{M}=0.246$ and $C_{B}=0.125$. Furthermore, because the spatial footprint of our survey is different in $B$ and $M$, we only have color information over a subset of our survey area. In regions where we do not having imaging in both filters, we use a $B-V$ color of 0.7 (similar to the color of the diffuse ICL; Rudick et al. 2010) in the photometric solution to calculate surface brightnesses in $B$ and $V$. If the intrinsic colors of objects are much bluer than this (for example, near spiral galaxies or galactic cirrus, where the colors will be a bluer $B-V \sim 0.4$ or so), this will systematically underestimate the surface brightness by about $0.1 \mathrm{mag} \mathrm{arcsec}^{-2}$ at most. At the faint surface brightnesses we are most interested in $\left(\mu_{V}>27\right)$, the uncertainty in sky will begin to dominate over this systematic uncertainty in color. With these choices, our final photometric solution is given by:

$$
\begin{aligned}
& m_{B}=-2.5 \log _{10}\left(I_{B}\right)+0.125(B-V)+28.973 \\
& m_{V}=-2.5 \log _{10}\left(I_{M}\right)+0.246(B-V)+28.606
\end{aligned}
$$

where $I_{B}$ and $I_{M}$ are the pixel values (in ADU) in the $B$ and $M$ mosaics, respectively. 


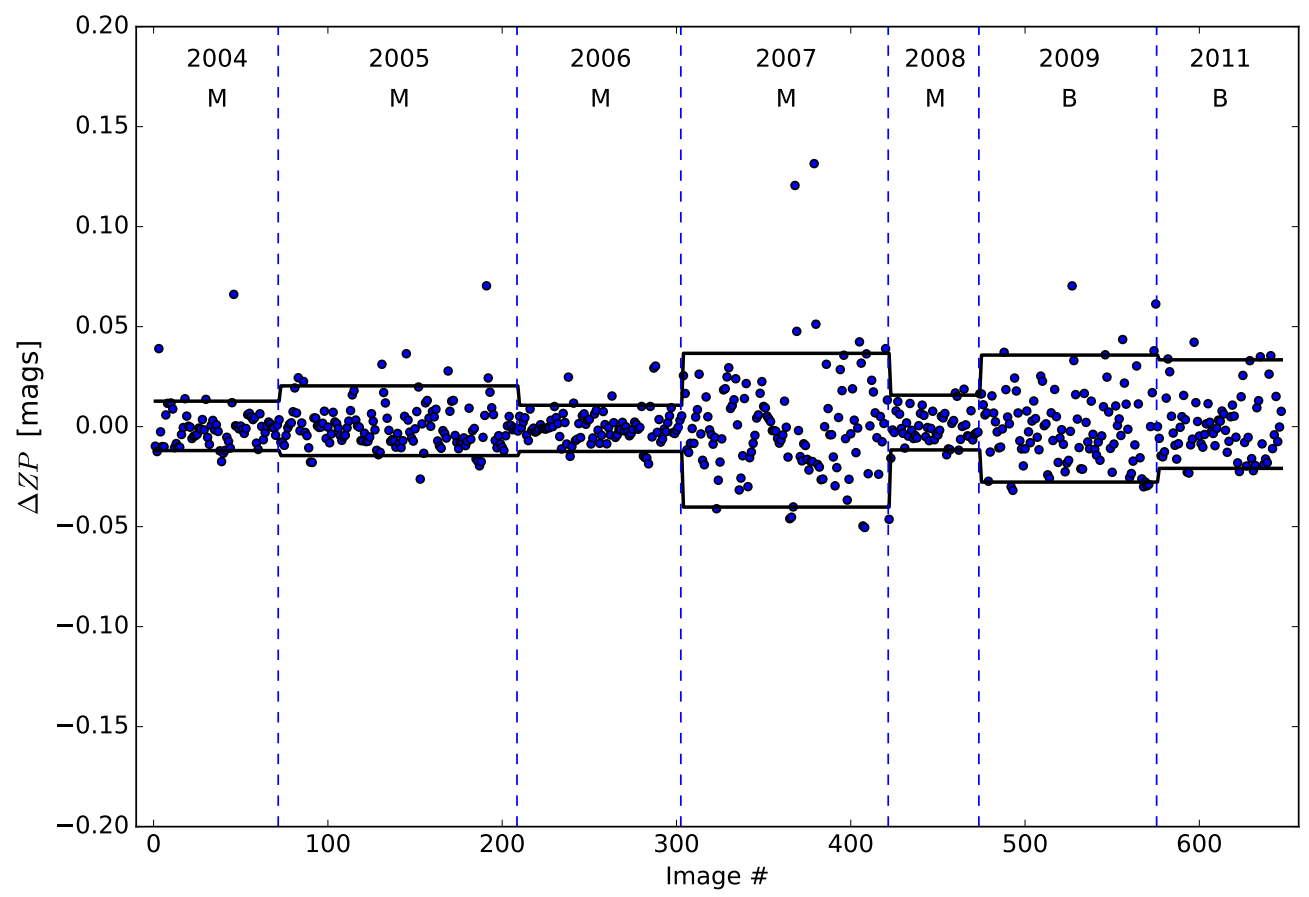

Figure 13. Frame-to-frame variation in photometric zeropoint, after removal of airmass term and nightly zeropoint offset. Different seasons are delineated by dashed vertical lines, and the solid horizontal lines show the $95 \%$ range in each season.

\section{REFERENCES}

Ahn, C. P., Alexandroff, R., Allende Prieto, C., et al. 2012, ApJS, 203, 21

Alam, S., Albareti, F. D., Allende Prieto, C., et al. 2015, ApJS, 219, 12

Arnaboldi, M., Freeman, K. C., Mendez, R. H., et al. 1996, ApJ, 472,145

Arnaboldi, M., Ventimiglia, G., Iodice, E., Gerhard, O., \& Coccato, L. 2012, A\&A, 545, A37

Arnaboldi, M., Aguerri, J. A. L., Napolitano, N. R., et al. 2002, AJ, 123, 760

Arnaboldi, M., \& Gerhard, O. 2010, Highlights of Astronomy, 15, 97

Arnaboldi, M., Gerhard, O., Aguerri, J. A. L., et al. 2004, ApJL, 614, L33

Arp, H., \& Bertola, F. 1969, Astrophys. Lett., 4, 23

Arrigoni Battaia, F., Gavazzi, G., Fumagalli, M., et al. 2012, A\&A, 543, A112

Böhringer, H., Briel, U. G., Schwarz, R. A., et al. 1994, Nature, 368,828

Bakos, J., Trujillo, I., \& Pohlen, M. 2008, ApJL, 683, L103

Barbary, K., Aldering, G., Amanullah, R., et al. 2012, ApJ, 745,

Barbosa, C. E., Arnaboldi, M., Coccato, L., et al. 2016, A\&A, 589, A139

Bautz, L. P., \& Morgan, W. W. 1970, ApJL, 162, L149

Beasley, M. A., Romanowsky, A. J., Pota, V., et al. 2016, ApJL, 819, L20

Becker, R. H., White, R. L., \& Helfand, D. J. 1995, ApJ, 450, 559

Bekki, K., Couch, W. J., Drinkwater, M. J., \& Shioya, Y. 2003, MNRAS, 344, 399

Bender, R., \& Surma, P. 1992, A\&A, 258, 250

Bender, R., Kormendy, J., Cornell, M. E., \& Fisher, D. B. 2015 , ApJ, 807, 56

Bender, R., Surma, P., Doebereiner, S., Moellenhoff, C., \& Madejsky, R. 1989, A\&A, 217, 35

Bernstein, G. M., Nichol, R. C., Tyson, J. A., Ulmer, M. P., \& Wittman, D. 1995, AJ, 110, 1507
Bianchi, S., Giovanardi, C., Smith, M. W. L., et al. 2016, arXiv:1609.05941

Binggeli, B., Popescu, C. C., \& Tammann, G. A. 1993, A\&AS, 98,275

Binggeli, B., Sandage, A., \& Tammann, G. A. 1985, AJ, 90, 1681

Binggeli, B., Tammann, G. A., \& Sandage, A. 1987, AJ, 94, 251

Blom, C., Forbes, D. A., Brodie, J. P., et al. 2012, MNRAS, 426, 1959

Blom, C., Forbes, D. A., Foster, C., Romanowsky, A. J., \& Brodie, J. P. 2014, MNRAS, 439, 2420

Blom, C., Spitler, L. R., \& Forbes, D. A. 2012, MNRAS, 420, 37

Bogdán, Á., Forman, W. R., Zhuravleva, I., et al. 2012, ApJ, 753 140

Boselli, A., Boissier, S., Heinis, S., et al. 2011, A\&A, 528, A107

Brandt, T. D., \& Draine, B. T. 2012, ApJ, 744, 129

Bruzual, G., \& Charlot, S. 2003, MNRAS, 344, 1000

Burke, C., Collins, C. A., Stott, J. P., \& Hilton, M. 2012 , MNRAS, 425, 2058

Burke, C., Hilton, M., \& Collins, C. 2015, MNRAS, 449, 2353

Busko, I. C. 1996, Astronomical Data Analysis Software and Systems V, 101, 139

Caldwell, N. 2006, ApJ, 651, 822

Capaccioli, M., Spavone, M., Grado, A., et al. 2015, A\&A, 581, A10

Cappellari, M., Emsellem, E., Krajnović, D., et al. 2011, MNRAS, 413, 813

Carter, D., \& Dixon, K. L. 1978, AJ, 83, 574

Castro-Rodriguéz, N., Arnaboldi, M., Aguerri, J. A. L., et al. 2009, A\&A, 507, 621

Ciardullo, R., Mihos, J. C., Feldmeier, J. J., Durrell, P. R., \& Sigurdsson, S. 2004, Recycling Intergalactic and Interstellar Matter, 217, 88

Condon, J. J., Cotton, W. D., Greisen, E. W., et al. 1998, AJ, 115,1693

Conroy, C., Wechsler, R. H., \& Kravtsov, A. V. 2007, ApJ, 668, 826

Contini, E., De Lucia, G., Villalobos, Á., \& Borgani, S. 2014 MNRAS, 437, 3787

Cooper, A. P., Gao, L., Guo, Q., et al. 2015, MNRAS, 451, 2703 
Cortés, J. R., Kenney, J. D. P., \& Hardy, E. 2008, ApJ, 683, 78-93

Cortese, L., Bendo, G. J., Isaak, K. G., Davies, J. I., \& Kent, B. R. 2010, MNRAS, 403, L26

Cortese, L., Gavazzi, G., Boselli, A., \& Iglesias-Paramo, J. 2004, A\&A, 416, 119

Cui, W., Murante, G., Monaco, P., et al. 2014, MNRAS, 437, 816

D'Onghia, E., Sommer-Larsen, J., Romeo, A. D., et al. 2005, ApJL, 630, L109

D’Onghia, E., Besla, G., Cox, T. J., \& Hernquist, L. 2009, Nature, 460, 605

D'Souza, R., Kauffman, G., Wang, J., \& Vegetti, S. 2014, MNRAS, 443, 1433

Da Rocha, C., \& Mendes de Oliveira, C. 2005, MNRAS, 364, 1069

Da Rocha, C., Ziegler, B. L., \& Mendes de Oliveira, C. 2008, MNRAS, 388, 1433

Davies, J. I., Baes, M., Bendo, G. J., et al. 2010, A\&A, 518, L48

Davies, J. I., Bianchi, S., Cortese, L., et al. 2012, MNRAS, 419, 3505

De Lucia, G., \& Blaizot, J. 2007, MNRAS, 375, 2

de Vaucouleurs, G., \& de Vaucouleurs, A. 1970, Astrophys. Lett., 5,219

de Vaucouleurs, G. 1961, ApJS, 6, 213

Dilday, B., Bassett, B., Becker, A., et al. 2010, ApJ, 715, 1021

Doherty, M., Arnaboldi, M., Das, P., et al. 2009, A\&A, 502, 771

Dolag, K., Murante, G., \& Borgani, S. 2010, MNRAS, 405, 1544

Donahue, M., Smith, B. J., \& Stocke, J. T. 2002, AJ, 123, 1922

Duc, P.-A., Brinks, E., Springel, V., et al. 2000, AJ, 120, 1238

Duc, P.-A., Cuillandre, J.-C., Karabal, E., et al. 2015, MNRAS, 446, 120

Durbala, A., del Olmo, A., Yun, M. S., et al. 2008, AJ, 135, 130

Durrell, P. R., Côté, P., Peng, E. W., et al. 2014, ApJ, 794, 103

Durrell, P. R., Ciardullo, R., Feldmeier, J. J., Jacoby, G. H., \& Sigurdsson, S. 2002, ApJ, 570, 119

Emsellem, E., Cappellari, M., Krajnović, D., et al. 2011, MNRAS, 414, 888

Feldmeier, J. J., Ciardullo, R., Jacoby, G. H., \& Durrell, P. R. 2004, ApJ, 615, 196

Feldmeier, J. J., Mihos, J. C., Morrison, H. L., et al. 2004, ApJ, 609,617

Feldmeier, J. J., Mihos, J. C., Morrison, H. L., Rodney, S. A., \& Harding, P. 2002, ApJ, 575, 779

Ferguson, H. C., Tanvir, N. R., \& von Hippel, T. 1998, Nature, 391,461

Ferrarese, L., Côté, P., Cuillandre, J.-C., et al. 2012, ApJS, 200, 4

Ferrarese, L., Côté, P., Sánchez-Janssen, R., et al. 2016, ApJ, 824, 10

Finkbeiner, D. P., Davis, M., \& Schlegel, D. J. 1999, ApJ, 524, 867

Frei, Z., \& Gunn, J. E. 1994, AJ, 108, 1476

Gal-Yam, A., Maoz, D., Guhathakurta, P., \& Filippenko, A. V. 2003, AJ, 125, 1087

Gavazzi, G., Boselli, A., Scodeggio, M., Pierini, D., \& Belsole, E. 1999, MNRAS, 304, 595

Gerhard, O., Arnaboldi, M., Freeman, K. C., et al. 2007, A\&A, 468,815

Gerhard, O., Arnaboldi, M., Freeman, K. C., \& Okamura, S. 2002, ApJL, 580, L121

Giallongo, E., Menci, N., Grazian, A., et al. 2014, ApJ, 781, 24

Giovanelli, R., Haynes, M. P., Kent, B. R., et al. 2005, AJ, 130, 2598

Girardi, M., Biviano, A., Giuricin, G., Mardirossian, F., \& Mezzetti, M. 1995, ApJ, 438, 527

Gonzalez, A. H., Zabludoff, A. I., \& Zaritsky, D. 2005, ApJ, 618, 195

Gonzalez, A. H., Zaritsky, D., \& Zabludoff, A. I. 2007, ApJ, 666, 147

Gregg, M. D., \& West, M. J. 1998, Nature, 396, 549

Guennou, L., Adami, C., Da Rocha, C., et al. 2012, A\&A, 537, A64

Guhathakurta, P., \& Tyson, J. A. 1989, ApJ, 346, 773

Guo, Q., White, S., Boylan-Kolchin, M., et al. 2011, MNRAS, 413,101

Hester, J. A., Seibert, M., Neill, J. D., et al. 2010, ApJL, 716, L14

Hibbard, J. E., Guhathakurta, P., van Gorkom, J. H., \& Schweizer, F. 1994, AJ, 107, 67

Hummel, E., Kotanyi, C. G., \& van Gorkom, J. H. 1986, A\&A, 155,161
Ienaka, N., Kawara, K., Matsuoka, Y., et al. 2013, ApJ, 767, 80

Impey, C., Bothun, G., \& Malin, D. 1988, ApJ, 330, 634

Irwin, J. A., \& Sarazin, C. L. 1996, ApJ, 471, 683

Janowiecki, S., Mihos, J. C., Harding, P., et al. 2010, ApJ, 715,

972

Jedrzejewski, R. I. 1987, MNRAS, 226, 747

Jerjen, H., Binggeli, B., \& Barazza, F. D. 2004, AJ, 127, 771

Jordán, A., Côté, P., West, M. J., et al. 2004, AJ, 127, 24

Katsiyannis, A. C., Kemp, S. N., Berry, D. S., \& Meaburn, J. 1998, A\&AS, 132, 387

Kelson, D. D., Zabludoff, A. I., Williams, K. A., et al. 2002, ApJ, 576,720

Kenney, J. D. P., Geha, M., Jáchym, P., et al. 2014, ApJ, 780, 119

Khochfar, S., \& Burkert, A. 2005, MNRAS, 359, 1379

Kim, D.-W., Fabbiano, G., \& Trinchieri, G. 1992, ApJ, 393, 134

Kim, S., Rey, S.-C., Jerjen, H., et al. 2014, ApJS, 215, 22

Koda, J., Yagi, M., Yamanoi, H., \& Komiyama, Y. 2015, ApJL, 807, L2

Kormendy, J., Fisher, D. B., Cornell, M. E., \& Bender, R. 2009,

ApJS, 182, 216

Kraft, R. P., Forman, W. R., Jones, C., et al. 2011, ApJ, 727, 41

Krick, J. E., \& Bernstein, R. A. 2007, AJ, 134, 466

Krisciunas, K., Semler, D. R., Richards, J., et al. 2007, PASP, 119,687

Leach, R. W., \& Low, F. J. 2000, Proc. SPIE, 4008, 337

Lee, H., Richer, M. G., \& McCall, M. L. 2000, ApJL, 530, L17

Lee, M. G., Park, H. S., \& Hwang, H. S. 2010, Science, 328, 334

Lelli, F., Duc, P.-A., Brinks, E., et al. 2015, A\&A, 584, A113

Lin, Y.-T., \& Mohr, J. J. 2004, ApJ, 617, 879

Liu, C., Peng, E. W., Côté, P., et al. 2015, ApJ, 812, 34

Liu, Y., Zhou, X., Ma, J., et al. 2005, AJ, 129, 2628

Longobardi, A., Arnaboldi, M., Gerhard, O., \& Mihos, J. C. 2015, A\&A, 579, L3

Longobardi, A., Arnaboldi, M., Gerhard, O., \& Hanuschik, R. 2015, A\&A, 579, A135

Low, F. J., Young, E., Beintema, D. A., et al. 1984, ApJL, 278, L19

Malin, D. 1994, "IAU Symposium 161: Astronomy from Wide-Field Imaging," eds. H. T. MacGillivray, E. B. Thomson,

B. M. Lasker, I. N. Reid, D. F. Malin, R. M. West \& H.

Lorenz. (Dordrecht: Kluwer), 567

Malin, D. F. 1979, Nature, 277, 279

Malin, D., \& Hadley, B. 1999, "Galaxy Dynamics," eds. D. R. Merritt, M. Valluri, \& J. A. Sellwood (San Francisco, CA:

Astronomical Society of the Pacific), 445

Martel, H., Barai, P., \& Brito, W. 2012, ApJ, 757, 48

McLaughlin, D. E. 1999, ApJL, 512, L9

McNamara, B. R., Sancisi, R., Henning, P. A., \& Junor, W. 1994, AJ, 108, 844

Mei, S., Blakeslee, J. P., Côté, P., et al. 2007, ApJ, 655, 144

Melnick, J., Hoessel, J., \& White, S. D. M. 1977, MNRAS, 180, 207

Mihos, C. 2015, IAU General Assembly, 22, 2247903

Mihos, J. C. 2004, "Clusters of Galaxies: Probes of Cosmological Structure and Galaxy Evolution," eds. J.S. Mulchaey, A. Dressler, \& A. Oemler (Cambridge University Press), 277

Mihos, J. C., Durrell, P. R., Ferrarese, L., et al. 2015, ApJL, 809, L21

Mihos, J. C., Harding, P., Feldmeier, J., \& Morrison, H. 2005, ApJL, 631, L41

Mihos, J. C., Harding, P., Rudick, C. S., \& Feldmeier, J. J. 2013, ApJL, 764, L20

Mihos, J. C., Harding, P., Spengler, C. E., Rudick, C. S., \& Feldmeier, J. J. 2013, ApJ, 762, 82

Mihos, J. C., Janowiecki, S., Feldmeier, J. J., Harding, P., \& Morrison, H. 2009, ApJ, 698, 1879

Miville-Deschênes, M.-A., \& Lagache, G. 2005, ApJS, 157, 302

Montes, M., \& Trujillo, I. 2014, ApJ, 794, 137

Montes, M., Trujillo, I., Prieto, M. A., \& Acosta-Pulido, J. A. 2014, MNRAS, 439, 990

Moore, B., Katz, N., Lake, G., Dressler, A., \& Oemler, A. 1996, Nature, 379, 613

Morrison, H. L., Miller, E. D., Harding, P., Stinebring, D. R., \& Boroson, T. A. 1997, AJ, 113, 2061

Murante, G., Arnaboldi, M., Gerhard, O., et al. 2004, ApJL, 607, L83 
Murante, G., Giovalli, M., Gerhard, O., et al. 2007, MNRAS, 377, 2

Naab, T., Jesseit, R., \& Burkert, A. 2006, MNRAS, 372, 839

Nassau, J. J. 1945, ApJ, 101, 275

Neill, J. D., Shara, M. M., \& Oegerle, W. R. 2005, ApJ, 618, 692

Neugent, K. F., \& Massey, P. 2010, PASP, 122, 1246

Ohyama, Y., \& Hota, A. 2013, ApJL, 767, L29

Okamura, S., Yasuda, N., Arnaboldi, M., et al. 2002, PASJ, 54, 883

Oosterloo, T., \& van Gorkom, J. 2005, A\&A, 437, L19

Patat, F. 2008, A\&A, 481, 575

Peng, E. W., Ferguson, H. C., Goudfrooij, P., et al. 2011, ApJ, 730,23

Peng, E. W., \& Lim, S. 2016, ApJL, 822, L31

Pfeffer, J., \& Baumgardt, H. 2013, MNRAS, 433, 1997

Pompea, S. M., Shepard, D. F., \& Anderson, S. 1989, Proc. SPIE 967, 236

Presotto, V., Girardi, M., Nonino, M., et al. 2014, A\&A, 565, A126

Puchwein, E., Springel, V., Sijacki, D., \& Dolag, K. 2010, MNRAS, 406, 936

Purcell, C. W., Bullock, J. S., \& Zentner, A. R. 2007, ApJ, 666, 20

Rines, K., \& Geller, M. J. 2008, AJ, 135, 1837

Romanowsky, A. J., Strader, J., Brodie, J. P., et al. 2012, ApJ, 748, 29

Rudick, C. S., Mihos, J. C., Frey, L. H., \& McBride, C. K. 2009, ApJ, 699, 1518

Rudick, C. S., Mihos, J. C., Harding, P., et al. 2010, ApJ, 720, 569

Rudick, C. S., Mihos, J. C., \& McBride, C. 2006, ApJ, 648, 936

Rudick, C. S., Mihos, J. C., \& McBride, C. K. 2011, ApJ, 732, 48

Sand, D. J., Graham, M. L., Bildfell, C., et al. 2011, ApJ, 729, 142

Sandage, A. 1976, AJ, 81, 954

Sandin, C. 2014, A\&A, 567, A97

Sandin, C. 2015, A\&A, 577, A106

Seigar, M. S., Graham, A. W., \& Jerjen, H. 2007, MNRAS, 378, 1575

Shapley, H., \& Ames, A. 1930, Harvard College Observatory Bulletin, 873, 1

Slater, C. T., Harding, P., \& Mihos, J. C. 2009, PASP, 121, 1267

Slinglend, K., Batuski, D., Miller, C., et al. 1998, ApJS, 115, 1

Smith, H. A. 1981, AJ, 86, 998

Solanes, J. M., Sanchis, T., Salvador-Solé, E., Giovanelli, R., \& Haynes, M. P. 2002, AJ, 124, 2440

Sommer-Larsen, J. 2006, MNRAS, 369, 958

Sommer-Larsen, J., Romeo, A. D., \& Portinari, L. 2005, MNRAS, 357,478
Struble, M. F. 1988, ApJL, 330, L25

Sun, M., Donahue, M., Roediger, E., et al. 2010, ApJ, 708, 946

Thuan, T. X., \& Kormendy, J. 1977, PASP, 89, 466

Toledo, I., Melnick, J., Selman, F., et al. 2011, MNRAS, 414, 602

Toomre, A., \& Toomre, J. 1972, ApJ, 178, 623

Tovmassian, H. M., \& Andernach, H. 2012, MNRAS, 427, 2047

Trujillo, I., \& Fliri, J. 2016, ApJ, 823, 123

Tutukov, A. V., \& Fedorova, A. V. 2011, Astronomy Reports, 55, 383

Urban, O., Werner, N., Simionescu, A., Allen, S. W., \&

Böhringer, H. 2011, MNRAS, 414, 2101

van Dokkum, P. G., Abraham, R., Merritt, A., et al. 2015, ApJL, $798, \mathrm{~L} 45$

Veneziani, M., Ade, P. A. R., Bock, J. J., et al. 2010, ApJ, 713, 959

Ventimiglia, G., Arnaboldi, M., \& Gerhard, O. 2011, A\&A, 528, A24

Vilchez-Gomez, R., Pello, R., \& Sanahuja, B. 1994, A\&A, 283, 37

Watkins, A. E., Mihos, J. C., Harding, P., \& Feldmeier, J. J.

2014, ApJ, 791, 38

Watkins, A. E., Mihos, J. C., \& Harding, P. 2016, ApJ, 826, 59

Watson, D. F., Berlind, A. A., \& Zentner, A. R. 2012, ApJ, 754, 90

Weil, M. L., Bland-Hawthorn, J., \& Malin, D. F. 1997, ApJ, 490, 664

Weil, M. L., \& Hernquist, L. 1996, ApJ, 460, 101

Weilbacher, P. M., Duc, P.-A., Fritze v. Alvensleben, U., Martin, P., \& Fricke, K. J. 2000, A\&A, 358, 819

Williams, B. F., Ciardullo, R., Durrell, P. R., et al. 2007, ApJ, 654,835

Williams, B. F., Ciardullo, R., Durrell, P. R., et al. 2007, ApJ, 656,756

Willman, B., Governato, F., Wadsley, J., \& Quinn, T. 2004, MNRAS, 355, 159

Witt, A. N., Mandel, S., Sell, P. H., Dixon, T., \& Vijh, U. P. 2008, ApJ, 679, 497-511

Yang, B., Zhu, J., \& Song, Y.-Y. 2002, Chinese J. Astron. Astrophys., 2, 474

Yasuda, N., Fukugita, M., \& Okamura, S. 1997, ApJS, 108, 417

York, D. G., Adelman, J., Anderson, J. E., Jr., et al. 2000, AJ, 120,1579

Yoshida, M., Yagi, M., Okamura, S., et al. 2002, ApJ, 567, 118

Zheng, Z., Thilker, D. A., Heckman, T. M., et al. 2015, ApJ, 800, 120

Zibetti, S., White, S. D. M., Schneider, D. P., \& Brinkmann, J.

2005, MNRAS, 358, 949 